\title{
Gravity as a four dimensional algebraic quantum field theory
}

\author{
GÁBOR Etesi
}

\begin{abstract}
Based on a family of indefinite unitary representations of the diffeomorphism group of an oriented smooth 4-manifold, a manifestly covariant 4 dimensional and non-perturbative algebraic quantum field theory formulation of gravity is exhibited. More precisely among the bounded linear operators acting on these representation spaces we identify algebraic curvature tensors hence a net of local quantum observables can be constructed from $C^{*}$-algebras generated by local curvature tensors and vector fields. This algebraic quantum field theory is extracted from structures provided by an oriented smooth 4-manifold only hence possesses a diffeomorphism symmetry. In this way classical general relativity exactly in 4 dimensions naturally embeds into a quantum framework.

Several Hilbert space representations of the theory are found. First a "tautological representation" of the limiting global $C^{*}$ algebra is constructed allowing to associate to any oriented smooth 4-manifold a von Neumann algebra in a canonical fashion. Secondly, influenced by the Dougan-Mason approach to gravitational quasilocal energy-momentum, we construct certain representations what we call "positive mass representations" with unbroken diffeomorphism symmetry. Thirdly, we also obtain "classical representaions" with spontaneously broken diffeomorphism symmetry corresponding to the classical limit of the theory which turns out to be general relativity.

Finally we observe that the whole family of "positive mass representations" comprise a 2 dimensional conformal field theory in the sense of G. Segal.
\end{abstract}

\section{Introduction}

The outstanding problem of modern theoretical physics is how to unify the obviously successful and mathematically consistent theory of general relativity with the obviously successful but yet mathematically problematic relativistic quantum field theory. It has been generally believed that these 
two fundamental pillars of modern theoretical physics conflict each other not only in the mathematical tools they use but even at a deep foundational level [12]: classical concepts of general relativity such as the space-time event, the light cone or the event horizon of a black hole are too "sharp" objects from a quantum theoretic viewpoint meanwhile relativistic quantum field theory is not background independent from the aspect of general relativity. We do not attempt here to survey the vast physical and even mathematical and philosophical literature created by the unification problem; we just mention that nowadays the two leading candidates expected to be capable for a sort of unification are string theory and loop quantum gravity. But surely there is still a long way ahead; nevertheless we have the conviction that one day the language of classical general relativity will sound familiar to quantum theorists and vice versa i.e., conceptual bridges must exist connecting the two theories.

In this note an effort has been made to embed classical general relativity into a quantum framework. This quantum framework is algebraic quantum field theory formulated by Haag-Kastler and others during the past decades, cf. [10]. Recently this language also appears to be suitable for formulating quantum field theory on curved space-time $[3,13]$ or even quantum gravity $[2]$.

In more detail we will do something very simple here. Namely using structures provided by an oriented smooth 4-manifold $M$ only, our overall guiding principle will be seeking unitary representations of the corresponding orientation-preserving diffeomorphism group $\operatorname{Diff}^{+}(M)$. There is a unique such representation via pullback on the incomplete space of sections of $\wedge^{2} M \otimes_{\mathbb{R}} \mathbb{C}$. However the natural scalar product on this space - namely the one given by integration of the wedge product of two 2 -forms - is indefinite hence cannot be used to complete the space of smooth 2-forms into a Hilbert space. Rather in struggling with the completion problem one comes up with a family of Hilbert spaces with a common non-degenerate indefinite Hermitian scalar product on them. The bare Hilbert spaces - i.e., not considered as $\operatorname{Diff}^{+}(M)$-modules - admit decompositions $\mathscr{H}^{+}(M) \oplus \mathscr{H}^{-}(M)$ into maximal definite orthogonal Hilbert subspaces $\mathscr{H}^{ \pm}(M)$ with respect to the indefinite scalar product. One can use this family of Hilbert spaces to discover an interesting $C^{*}$-algebra by exploring their spaces of bounded linear operators. It indeed comes as a surprise (at least to the author) that precisely in 4 dimensions among these operators one can recognize curvature tensors! This is because of the well-known fact that the curvature tensor $R_{g}$ of a pseudo-Riemannian 4-manifold $(M, g)$ can be viewed as a section of $\operatorname{End}\left(\wedge^{2} M \otimes_{\mathbb{R}} \mathbb{C}\right)$ i.e., gives rise to a linear operator acting on 
any $\mathscr{H}^{+}(M) \oplus \mathscr{H}^{-}(M)$. This permits to construct a net $\{U \mapsto \mathfrak{A}(U)\}_{U \subseteq M}$ whose local $C^{*}$-algebras are generated by bundle endomorphisms and $\overline{\bar{L}}$ ie derivatives. These local algebras are generalizations of the CCR algebra. The construction satisfies the naturally generalized Haag-Kastler axioms [10, pp. 105-107] leading to an algebraic quantum field theory in which Poincaré symmetry is replaced with full diffeomorphism symmetry (if the diffeomorphism group is regarded as the physical symmetry group of general relativity and not its gauge group). As a result classical general relativity effortlessly embeds into a quantum framework if one interprets classical curvature tensors as quantum observables. The appearence of the curvature tensor as a local quantum observable is reasonable even from the physical viewpoint: in local gravitational physics the metric tensor has no direct physical meaning only its curvature can cause local physical effects such as tidal forces. Moreover if one wishes, at least in principle, the metric i.e., the geometry locally can be reconstructed from its curvature (see e.g. [5, 9, 11, 14] and the references therein).

We also exhibit several Hilbert space representations of the theory carrying unitary representations of the diffeomorphism group. The first one is a "tautological representation" of the global algebra on itself allowing us to attach to $M$ a von Neumann algebra $\mathfrak{R}(M)$. The other ones deal with physics. A meaningful quantum field theory must exhibit stability i.e., "positive mass representations" of its local observables in the sense of Wigner. In our case this directly leads to the long-standing problem of gravitational mass [18]. It is quite interesting that the Gelfand-Naimark-Segal construction in the theory of $C^{*}$-algebras and quasilocal energy-momentum constructions [18] in general relativity naturally meet up here because immersed surfaces in $M$ provide us with both $C^{*}$-algebra representations and Dougan-Mason-like quasilocal quantities [7]. More precisely our quasilocal energy-momenta and masses stem from quasilocal translations along immersed surfaces in $M$ with a choice of a complex structure on them. However the whole construction is expected to be independent of this choice leading to the by-now classical observation of Witten [21] that in fact one has to deal with a conformal field theory on these surfaces. We identify this theory: its spaces of conformal blocks are the Clifford algebras generated by finite energy meromorphic sections of certain unitary holomorphic vector bundles on punctured Riemannian surfaces. Apart from these quantum representations, "classical representations" corresponding to the classical limit of the theory also exist. Indeed, unlike in the previous two cases, in these representations the diffeomorphism symmetry spontaneously breaks down to a finite dimensional Lie subgroup provided by the isometry group of an emergent metric $g$ on 
$M$; hence a causal structure can be constructed on $M$ as well. Therefore classical general relativity is recovered again at the representation theoretic level. The emergent metric distinguishes a canonically split Hilbert space $\mathscr{H}^{+}(M) \oplus \mathscr{H}^{-}(M)$ provided by metric (anti)self-duality leading to a splitting of the Hilbert space of the corresponding "classical representation", too. The natural quantum observable provided by the curvature $R_{g}$ of the metric in this representation obeys the splitting if and only if $g$ is a vacuum metric.

However our algebraic quantum field theory itself lacks any causal structure in general as an unavoidable consequence of its vast diffeomorphism symmetry. ${ }^{1}$ The causal future $J^{+}(p) \subset M$ of an event $p \in M$ in space-time is by definition the union of all future-inextendible worldlines of particles departing from $p$ and moving forward in time locally not exceeding the speed of light. The causal past $J^{-}(p)$ is defined similarly. The collection of these subsets of space-time generates a special topology on $M$ in the strict mathematical sense. The Lorentzian metric is a mathematical fusion of the geometry of $M$ identified with a Riemannian metric and the causal structure of $M$ identified with this topology. But from this operational description of causality it is clear that the construction of a causal structure refers to not only gravity but other entities of physical reality as well which are moreover quite classical: pointlike particles, electromagnetic waves, time, etc. However they cannot appear for instance in a vacuum space-time considered in the strict sense. Very strictly speaking even the interpretation of a space-time point as a "physical event" fails in an empty space-time. Therefore we are convinced that causality cannot be a fundamental ingredient of a classical hence even of a quantum description of pure gravity if it is a diffeomorphisminvariant quantum field theory. As a technical consequence we will prefer to use Riemannian metrics in this note (although emphasize that mathematically all conclusions hold for Lorentzian metrics as well). To summarize: from our standpoint causality is an emergent statistical phenomenon created by the highly complex interaction of gravity and matter. Consequently in order to recover it first we should be able to break down the diffeomorphism symmetry and distinguish pure gravity from matter.

This note is organized as follows. In Section 2 we construct natural indefinite unitary representations of orientation-preserving diffeomorphisms of an oriented 4-manifold. Then we extract a unique $C^{*}$-algebra out of these representation spaces. We identify its "classical part" with Einstein manifolds. In Section 3 we introduce an algebraic quantum field theory and in

\footnotetext{
${ }^{1}$ This is in accordance with recent speculations on Lorentz symmetry violations for instance in extreme high energy cosmic processes, for a review cf. e.g. [4].
} 
Theorems 3.1 and 3.2 and 3.3 we construct certain representations of its algebras of local observables what we call "a tautological representation", "positive mass representations" and "classical representations" respectively. In Section 4 we bunch the positive mass representations together into a conformal field theory.

Acknowledgement. The author is grateful to M.J. Dupré, I. Ojima, L.B. Szabados and P. Vrana for the stimulating discussions and to the Alfréd Rényi Institute of Mathematics for their hospitality. This work was supported by OTKA grant No. NK81203 (Hungary).

\section{The $C^{*}$-algebra of an oriented smooth 4-manifold}

Let $M$ be a connected orientable smooth 4-manifold, possibly non-closed (i.e., it can be non-compact and-or with non-empty boundary). Fix an orientation on $M$. Given only these data at our disposal it is already meaningful to talk about the group of its orientation-preserving diffeomorphisms $\operatorname{Diff}^{+}(M)$. Our overall guiding principle simply will be a search for unitary representations of $\operatorname{Diff}^{+}(M)$. A bunch of representations arise in a geometric way as follows. Consider $T^{(r, s)} M \otimes_{\mathbb{R}} \mathbb{C}$, the bundle of complexified $(r, s)$-type tensors with the associated vector spaces $C_{c}^{\infty}\left(M ; T^{(r, s)} M \otimes_{\mathbb{R}} \mathbb{C}\right)$ of their compactly supported smooth complexified sections. Then the group $\operatorname{Diff}^{+}(M)$ acts from the left via pushforward on $C_{c}^{\infty}\left(M ; T^{(r, 0)} M \otimes_{\mathbb{R}} \mathbb{C}\right)$ for all $r \in \mathbb{N}$ while from the right via pullback on $C_{c}^{\infty}\left(M ; T^{(0, s)} M \otimes_{\mathbb{R}} \mathbb{C}\right)$ for all $s \in \mathbb{N}$. However these representations are typically not unitary because the underlying vector spaces do not carry extra structures in a natural way.

The only exception is the $2^{\text {nd }}$ exterior power $\wedge^{2} M \subset T^{(0,2)} M$ of the cotangent bundle with the corresponding space of sections $C_{c}^{\infty}\left(M ; \wedge^{2} M \otimes_{\mathbb{R}}\right.$ $\mathbb{C})=: \Omega_{c}^{2}(M ; \mathbb{C})$, the space of complexified smooth 2 -forms with compact support. Indeed, this vector space has a natural non-degenerate Hermite scalar product $\langle\cdot, \cdot\rangle_{L^{2}(M)}: \quad \Omega_{c}^{2}(M ; \mathbb{C}) \times \Omega_{c}^{2}(M ; \mathbb{C}) \rightarrow \mathbb{C}$ given by integration on oriented smooth manifolds; more precisely for $\alpha, \beta \in \Omega_{c}^{2}(M ; \mathbb{C})$ put

$$
\langle\alpha, \beta\rangle_{L^{2}(M)}:=\int_{M} \bar{\alpha} \wedge \beta
$$

(complex conjugate-linear in its first variable). Note however that this scalar product is indefinite: an unavoidable fact which plays a key role in our considerations ahead. Consequently this scalar product cannot be used to complete $\Omega_{c}^{2}(M ; \mathbb{C})$ into a Hilbert space. Instead with respect to $(1)$ there is 
a non-unique direct sum decomposition

$$
\Omega_{c}^{2}(M ; \mathbb{C})=\Omega_{c}^{+}(M ; \mathbb{C}) \oplus \Omega_{c}^{-}(M ; \mathbb{C})
$$

with the property that they are maximal definite orthogonal subspaces i.e., $\pm\left.\langle\cdot, \cdot\rangle_{L^{2}(M)}\right|_{\Omega_{c}^{ \pm}(M ; \mathbb{C})}: \Omega_{c}^{ \pm}(M ; \mathbb{C}) \times \Omega_{c}^{ \pm}(M ; \mathbb{C}) \rightarrow \mathbb{C}$ are both positive definite moreover $\Omega_{c}^{+}(M ; \mathbb{C}) \perp_{L^{2}(M)} \Omega_{c}^{-}(M ; \mathbb{C})$. Therefore these restricted scalar products can be used to complete $\Omega_{c}^{ \pm}(M ; \mathbb{C})$ into separable Hilbert spaces $\mathscr{H}^{ \pm}(M)$ respectively. That is, starting with an $M$ we can make $\Omega_{c}^{2}(M ; \mathbb{C})$ complete only in non-canonical ways as follows. The possible completions form a family and any member of this family consists of a particular direct sum Hilbert space $\mathscr{H}^{+}(M) \oplus \mathscr{H}^{-}(M)$ (with its particular non-degenerate positive definite scalar product $(\alpha, \beta)_{L^{2}(M)}:=$ $\left.\left\langle\alpha^{+}, \beta^{+}\right\rangle_{L^{2}(M)}-\left\langle\alpha^{-}, \beta^{-}\right\rangle_{L^{2}(M)}\right)$ and a common indefinite scalar product

$$
\langle\cdot, \cdot\rangle_{L^{2}(M)}: \mathscr{H}^{+}(M) \oplus \mathscr{H}^{-}(M) \times \mathscr{H}^{+}(M) \oplus \mathscr{H}^{-}(M) \longrightarrow \mathbb{C}
$$

induced by (1) such that:

$$
\left\{\begin{aligned}
\mathscr{H}^{+}(M) \perp_{L^{2}(M)} \mathscr{H}^{-}(M) & \\
\langle\cdot, \cdot\rangle_{L^{2}(M)} \mid \mathscr{H}^{ \pm}(M) & : \mathscr{H}^{ \pm}(M) \times \mathscr{H}^{ \pm}(M) \\
& \longrightarrow \mathbb{C} \text { are positive or negative definite, respectively. }
\end{aligned}\right.
$$

Moreover any $\left(\mathscr{H}^{+}(M) \oplus \mathscr{H}^{-}(M),\langle\cdot, \cdot\rangle_{L^{2}(M)}\right)$ carries a representation of $\operatorname{Diff}^{+}(M)$ from the right given by the unique continuous extension of the pullback of 2-forms: $\omega \mapsto f^{*} \omega$ for $\omega \in \Omega_{c}^{2}(M ; \mathbb{C})$ and $f \in \operatorname{Diff}^{+}(M)$. It is easy to check that these operators are unitary with respect to (2) and operators corresponding to compactly supported diffeomorphisms are also bounded with respect to the operator norm induced by the particular Hilbert space norm on $\mathscr{H}^{+}(M) \oplus \mathscr{H}^{-}(M)$. Note that a priori representations on different completions are not unitary equivalent.

These representations have the following immediate properties:

Lemma 2.1. Consider the indefinite unitary reprsentation of $\operatorname{Diff}^{+}(M)$ from the right on any particular $\left(\mathscr{H}^{+}(M) \oplus \mathscr{H}^{-}(M),\langle\cdot, \cdot\rangle_{L^{2}(M)}\right)$ constructed above.

(i) A vector $v \in \mathscr{H}^{+}(M) \oplus \mathscr{H}^{-}(M)$ satisfies $f^{*} v=v$ for all $f \in \operatorname{Diff}^{+}(M)$ if and only if $v=0$ ("no vacuum"); 
(ii) The closed subspaces $\mathscr{B}(M) \subseteq \mathscr{Z}(M) \subset \mathscr{H}^{+}(M) \oplus \mathscr{H}^{-}(M)$ generated by exact or closed 2-forms respectively are invariant under the action of $\operatorname{Diff}^{+}(M)$.

Proof. (i) Assume that there exists an element $0 \neq v \in \mathscr{H}^{+}(M) \oplus \mathscr{H}^{-}(M)$ stabilized by the whole $\operatorname{Diff}^{+}(M)$. Consider a 1-parameter subgroup $\left\{f_{t}\right\}_{t \in \mathbb{R}} \in \operatorname{Diff}^{+}(M)$ such that $f_{0}=\operatorname{Id}_{M}$ and let $X$ be the vector field on $M$ generating this subgroup. Differentiating the equation $f_{t}^{*} v=v$ with respect to $t \in \mathbb{R}$ at $t=0$ we obtain $L_{X} v=0$ (in the weak sense) where $L_{X}$ is the Lie derivative by $X$. Since an arbitrary compactly supported vector field generates a 1-parameter subgroup of $\operatorname{Diff}^{+}(M)$ we obtain that in fact $v=0$, a contradiction.

(ii) The statement readily follows by naturality of exterior differentiation i.e., $\mathrm{d}\left(f^{*} \varphi\right)=f^{*} \mathrm{~d} \varphi$ for all $f \in \operatorname{Diff}^{+}(M)$ and $\varphi \in \Omega_{c}^{k}(M ; \mathbb{C})$.

Remark. 1. We succeeded to construct a family of faithful, reducible, indefinite unitary representations of the diffeomorphism group out of the structures provided only by an orientable smooth 4-manifold. ${ }^{2}$ All of these representation spaces are split however such decompositions cannot hold as a $\operatorname{Diff}^{+}(M)$-module or in other words such decompositions break the diffeomorphism symmetry. The relevance of these splittings, as we will see shortly, is that the classical vacuum Einstein equation can be viewed as saying that there is a distinguished representation $\mathscr{H}^{+}(M) \oplus \mathscr{H}^{-}(M)$ on which the curvature is blockdiagonal i.e., respects the splitting. In general, starting only with an oriented smooth 4-manifold $M$ without extra structure, there is no way to associate a canonical non-split Hilbert space to $M$.

2. From the mathematical viewpoint in many important cases we do not loose topological information if we replace $M$ with any representation. Indeed, restricting $\Omega_{c}^{2}(M ; \mathbb{C})$ to closed forms and dividing by the exact ones we can pass to compactly supported cohomology $H_{c}^{2}(M ; \mathbb{C})$; then if $M$ admits a finite good cover Poincaré duality works and gives $H_{c}^{2}(M ; \mathbb{C}) \cong$ $\left(H^{2}(M ; \mathbb{C})\right)^{*}$. If we assume that $M$ is compact and simply connected then the singular cohomology $H^{2}(M ; \mathbb{Z})$ maps injectively into $H^{2}(M ; \mathbb{C})$ hence finally the scalar product (2) descends to the topological intersection form

$$
q_{M}: H^{2}(M ; \mathbb{Z}) \times H^{2}(M ; \mathbb{Z}) \longrightarrow H^{4}(M ; \mathbb{Z}) \cong \mathbb{Z}
$$

\footnotetext{
${ }^{2}$ In fact our construction so far works in any $4 k(k=1,2, \ldots)$ dimensions if the diffeomorphism group acts on $2 k$-forms. In $4 k+2$ dimensions (1) gives symplectic forms.
} 
of the underlying topological 4-manifold. However taking into account that by assumption $M$ has a smooth structure we can refer to Freedman's fundamental result [8] that $q_{M}$ uniquely determines the topology of $M$.

Now we proceed further and observe that in spite of this plethora of diffeomorphism group representations one can attach a unique $C^{*}$-algebra to an oriented smooth 4-manifold. However this $C^{*}$-algebra does not admit representations on the previous Hilbert spaces.

Lemma 2.2. Let $*$ be the adjoint operation on $\Omega_{c}^{2}(M ; \mathbb{C})$ for the indefinite scalar product (1). Consider the $*$-closed space $V:=\left\{A \in \operatorname{End}\left(\Omega_{c}^{2}(M ; \mathbb{C})\right) \mid\right.$ $\left.r\left(A^{*} A\right)<+\infty\right\}$ defined by the spectral radius

$$
r(B):=\sup _{\lambda \in \mathbb{C}}\left\{|\lambda| \mid B-\lambda \cdot \operatorname{Id}_{\Omega_{c}^{2}(M ; \mathbb{C})} \text { is not invertible }\right\} .
$$

Then $\sqrt{r}$ is a norm and the corresponding completion of $V$ renders $(V$, *) a unital $C^{*}$-algebra containing $\operatorname{Diff}^{+}(M)$. This $C^{*}$-algebra will be denoted by $\mathfrak{B}(M)$.

Proof. Our strategy to prove the lemma is as follows. Obviously $(V, *)$ is a *-algebra. Provided it can be equipped with a norm such that corresponding completion of $V$ improves $(V, *)$ to a $C^{*}$-algebra then knowing the uniqueness of the $C^{*}$-algebra norm this sought norm $[[\cdot]]$ on all $A \in V$ must look like $[[A]]^{2}=\left[\left[A^{*} A\right]\right]=r\left(A^{*} A\right)$. Therefore we want to see that the spectral radius gives a norm here.

Take any splitting $\Omega_{c}^{2}(M ; \mathbb{C})=\Omega_{c}^{+}(M ; \mathbb{C}) \oplus \Omega_{c}^{-}(M ; \mathbb{C})$ and the corresponding Hilbert space completion $\mathscr{H}^{+}(M) \oplus \mathscr{H}^{-}(M) \supset \Omega_{c}^{2}(M ; \mathbb{C})$. If $P^{ \pm}$: $\mathscr{H}^{+}(M) \oplus \mathscr{H}^{-}(M) \rightarrow \mathscr{H}^{ \pm}(M)$ are the orthogonal projections then put $J:=P^{+}-P^{-}$moreover let $\dagger$ denote the adjoint on $\mathscr{H}^{+}(M) \oplus \mathscr{H}^{-}(M)$. Then $J$ satisfies $A^{*}=J A^{\dagger} J$ and $J^{2}=\operatorname{Id}_{\mathscr{H}+(M) \oplus \mathscr{H}^{-}(M)}$ therefore $A^{\dagger}=$ $J A^{*} J$ as well. Recall that the operator norm is

$$
\|B\|=\sup _{v \neq 0} \frac{\|B v\|_{L^{2}(M)}}{\|v\|_{L^{2}(M)}}
$$

where $\|\cdot\|_{L^{2}(M)}$ comes from the positive definite scalar product $(\cdot, \cdot)_{L^{2}(M)}$ on $\mathscr{H}^{+}(M) \oplus \mathscr{H}^{-}(M)$. Since $\|J\|=1$ it readily follows from this definition that $\left\|J A^{*} J A\right\|=\left\|A^{*} A\right\|$. The adjoint $\dagger$ and the norm $\|\cdot\|$ are actually the $*$-operation and norm on the particular $C^{*}$-algebra of bounded linear operators on the particular Hilbert space $\mathscr{H}^{+}(M) \oplus \mathscr{H}^{-}(M)$. Therefore taking into account again the uniqueness of $C^{*}$-algebra norm we also have 
equalities $\|A\|^{2}=\left\|A^{\dagger} A\right\|=r\left(A^{\dagger} A\right)$. Additionally the spectral radius always satisfies $r(B)=\lim _{k \rightarrow+\infty}\left\|B^{k}\right\|^{\frac{1}{k}} \leqq\|B\|$ which is Gelfand's formula (cf. e.g. [15, $\S X I .149])$.

After these preparations we can embark upon the proof. On the one hand

$$
r\left(A^{*} A\right)=r\left(J A^{\dagger} J A\right) \leqq\left\|J A^{\dagger} J A\right\| \leqq\|A\|^{2} .
$$

On the other hand, for any $\varepsilon>0$ one can find a positive integer $k$ such that

$$
\begin{aligned}
\|A\|^{2}-\varepsilon & =r\left(A^{\dagger} A\right)-\varepsilon=r\left(J A^{*} J A\right)-\varepsilon \\
& \leqq\left\|\left(J A^{*} J A\right)^{k}\right\|^{\frac{1}{k}}=\left\|\left(A^{*} A\right)^{k}\right\|^{\frac{1}{k}} \leqq r\left(A^{*} A\right)+\varepsilon
\end{aligned}
$$

therefore, since $\varepsilon>0$ was arbitrary,

$$
\|A\|^{2} \leqq r\left(A^{*} A\right)
$$

We conclude that $r\left(A^{*} A\right)=\|A\|^{2}$ demonstrating that the spectral radius indeed provides us with a norm on $\Omega_{c}^{2}(M ; \mathbb{C})$. Consequently putting

$$
[[A]]:=\sqrt{r\left(A^{*} A\right)}
$$

we can complete $V$ with respect to this norm and enrich the $*$-algebra $(V, *)$ to a $C^{*}$-algebra $\mathfrak{B}(M)$.

Finally, since diffeomorphisms are unitary i.e., $\left(f^{*}\right)^{*}\left(f^{*}\right)=\operatorname{Id}_{\Omega_{c}^{2}(M ; \mathbb{C})}$ for all $f \in \operatorname{Diff}^{+}(M)$ we find $\left[\left[f^{*}\right]\right]=1$ which means that $f^{*} \in V \subset \mathfrak{B}(M)$ as stated.

Remark. From the proof of Lemma 2.2 we can also read off that although the individual Hilbert space completions $\mathscr{H}^{+}(M) \oplus \mathscr{H}^{-}(M) \supset \Omega_{c}^{2}(M ; \mathbb{C})$ might be unitary inequivalent, the induced operator norms on the common intersection of the individual algebras of bounded linear operators are not only equivalent as norms but even numerically equal. They are commonly given by (4).

For a relatively compact open subset $\emptyset \subseteq U \subseteq M$ a unital $C^{*}$-algebra $\mathfrak{B}(\emptyset) \subseteq$ $\mathfrak{B}(U) \subseteq \mathfrak{B}(M)$ is defined as the norm-completion of the $*$-closed space

$$
\left\{B \in \operatorname{End}\left(\Omega_{c}^{2}(M ; \mathbb{C})\right) \mid[[B]]<+\infty,\left[\left.B\right|_{\Omega_{c}^{2}(M \backslash U ; \mathbb{C})}, \operatorname{Diff}_{U}^{+}(M)\right]=0\right\}
$$

i.e., $\mathfrak{B}(U)$ consists of operators which commute on the subspace $\Omega_{c}^{2}(M \backslash$ $U ; \mathbb{C}) \subseteq \Omega_{c}^{2}(M ; \mathbb{C})$ with the subgroup $\operatorname{Diff}_{U}^{+}(M) \subseteq \operatorname{Diff}^{+}(M)$ consisting of 
all $U$-preserving diffeomorphisms. Since an operator commuting with all diffeomorphisms is proportional to the identity, $\mathfrak{B}(\emptyset) \cong \mathbb{C} \cdot 1$.

Consider the assignment $\{U \mapsto \mathfrak{B}(U)\}_{U \subseteq M}$ for all relatively compact open subsets. Taking into account that if $\overline{\bar{A}} \in \mathfrak{B}(U)$ then $\left.A\right|_{\Omega_{c}^{2}(M \backslash U ; \mathbb{C})}=$ $\mathbb{C} \operatorname{Id}_{\Omega_{c}^{2}(M \backslash U ; \mathbb{C})}$ and $\Omega_{c}^{2}(M \backslash V ; \mathbb{C}) \subseteq \Omega_{c}^{2}(M \backslash U ; \mathbb{C})$ if $U \subseteq V$ the embedding induces a unit-preserving injective homomorphism $e_{V}^{U}: \mathfrak{B}(U) \rightarrow \mathfrak{B}(V)$ of local $C^{*}$-algebras. This permits to define $\mathfrak{B}(U)$ for any open $\emptyset \subseteq U \subseteq M$ and $\mathfrak{B}(M)$ as the $C^{*}$-algebra direct (inductive) limit of these local algebras. Henceforth this assignment in fact defines a covariant functor from the category of open subsets of $M$ with inclusion into the category of unital $C^{*}$-alegbras with $*$-homomorphisms. However observe that if we consider the dual process namely the restriction then elements of these local algebras do not behave well because they lack the presheaf property in general.

As a consequence of the geometric origin of the global $C^{*}$-algebra $\mathfrak{B}(M)$, it has an interesting sub- $C^{*}$-algebra $\mathfrak{C}(M)$ if $M$ is compact. Indeed, consider the sheaf $\mathscr{C}_{M}$ over $M$ whose spaces of local sections $\mathscr{C}(U)$ over open subsets are algebras of local smooth bundle (i.e., fiberwise) morphisms

$$
C^{\infty}\left(U ; \operatorname{End}\left(\wedge^{2} U \otimes_{\mathbb{R}} \mathbb{C}\right)\right) \text { for all open } U \subseteq M .
$$

In contrast to general elements of $\mathfrak{B}(U)$, local sections in $\mathscr{C}(U)$ behave well under restriction due to their presheaf property; i.e., given two open subsets $U \subseteq V$ the restriction map induces a unit-preserving injective homomorphism $r_{U}^{V}: \mathscr{C}(V) \rightarrow \mathscr{C}(U)$ of algebras. Although $\mathfrak{B}(U)$ and $\mathscr{C}(U)$ are not related in general if $M$ happens to be compact the space $\mathscr{C}(M) \subset$ $\operatorname{End}\left(\Omega_{c}^{2}(M ; \mathbb{C})\right)$ of global sections can be completed with respect to $(4)$ to a unital $C^{*}$-algebra $\mathfrak{C}(M)$ and in this case there is an obvious embedding of unital $C^{*}$-algebras $\mathfrak{C}(M) \varsubsetneqq \mathfrak{B}(M)$.

Examples. The time has come to take a closer look of the various operator algebras $\mathfrak{B}(M)$ and $\mathscr{C}(M)$ (or $\mathfrak{C}(M)$ if $M$ is compact) associated to an oriented smooth 4-manifold $M$ emerging through unitary representations of its diffeomorphism group. We will see that especially in 4 dimensions these algebras admit rich physical interpretations as follows.

1. Let $(M, g)$ be a 4-dimensional Riemannian Einstein manifold i.e., assume that $g$ is a Riemannian metric on $M$ with Ricci tensor $r_{g}$ satisfying the vacuum Einstein equation $r_{g}=\Lambda_{M} g$ with a cosmological constant $\Lambda_{M} \in \mathbb{R}$. In this special situation the vast symmetry group of the original theory reduces to the stabilizer subgroup $\operatorname{Iso}^{+}(M, g) \varsubsetneqq \operatorname{Diff}^{+}(M)$ leaving the geometry $(M, g)$ unaffected. In this realm the Riemannian metric together with the orientation gives a Hodge operator $*_{g}: \wedge^{2} M \rightarrow \wedge^{2} M$ with $*_{g}^{2}=\operatorname{Id}_{\wedge^{2} M}$. 
This induces a usual real splitting

$$
\wedge^{2} M=\wedge^{+} M \oplus \wedge^{-} M
$$

It is well-known [17] but from our viewpoint is an interesting coincidence that in exactly 4 dimensions the full Riemannian curvature tensor can be regarded as a real linear bundle map $R_{g}: \wedge^{2} M \rightarrow \wedge^{2} M$ which as a bundle map decomposes i.e., over every point $x \in M$ decomposes like

$$
R_{g}=\left(\begin{array}{cc}
W_{g}^{+}+\frac{s_{g}}{12} & B_{g} \\
B_{g}^{*} & W_{g}^{-}+\frac{s_{g}}{12}
\end{array}\right)
$$

with respect to the splitting (5). Here the traceless symmetric maps $W_{g}^{ \pm}$: $\wedge^{ \pm} M \rightarrow \wedge^{ \pm} M$ are the (anti)self-dual parts of the Weyl tensor, the diagonal $s_{g}: \wedge^{2} M \rightarrow \wedge^{2} M$ is the scalar curvature while $B_{g}: \wedge^{+} M \rightarrow \wedge^{-} M$ is the traceless Ricci tensor together with its metric adjoint $B_{g}^{*}: \wedge^{-} M \rightarrow \wedge^{+} M$. Observe that the Einstein equation $r_{g}-\frac{1}{2} s_{g} g=8 \pi T-\Lambda_{M} g$ exactly says that

$$
\left\{\begin{array}{l}
B_{g}=8 \pi T_{0} \\
s_{g}=4 \Lambda_{M}-8 \pi \operatorname{tr}_{g} T
\end{array}\right.
$$

where $T_{0}$ is the traceless part of the energy-momentum tensor. The vacuum $T=0$ is equivalently characterized by the single condition $B_{g}=0$. Indeed, in this case always $T_{0}=0$ hence if $T \neq 0$ then matter is present only through its tracial part $\left(\frac{1}{4} \operatorname{tr}_{g} T\right) g$ moreover $\operatorname{tr}_{g} T$ is constant by the differential Bianchi identity. However by convention such a thing is not called as "matter" but rather is incorporated into the cosmological constant $\Lambda_{M}$. Consequently looking at the vacuum as being equivalent to the condition $B_{g}=0$, in the case of vacuum $R_{g} \in C^{\infty}\left(M ; \operatorname{End}\left(\wedge^{2} M\right)\right)$ obeys (5). The pointwise splitting above in addition yields the canonical decomposition

$$
\Omega_{c}^{2}(M ; \mathbb{C})=\Omega_{c}^{+}(M ; \mathbb{C}) \oplus \Omega_{c}^{-}(M ; \mathbb{C})
$$

of the space of 2-forms into (anti)self-dual forms which is the same as decomposing this space into mutually orthogonal maximal definite subspaces with respect to the scalar product (1). Therefore in the presence of a metric - which is a way to break the original symmetry group $\operatorname{Diff}^{+}(M)$ down to a smaller one - there is a splitting $\mathscr{H}^{+}(M) \oplus \mathscr{H}^{-}(M)$ preferred by the curvature $R_{g}$. Switching to our notation we conclude that $R_{g} \in \mathscr{C}(M)$ satisfies $R_{g}\left(\mathscr{H}^{ \pm}(M)\right) \subseteq \mathscr{H}^{ \pm}(M)$. Moreover by the usual symmetries of the curvature tensor $R_{g}$ is self-adjoint for $(2)$. For clarity we note that this action 
of for example $R_{g} \in \mathscr{C}(M)$ on $\mathscr{H}^{+}(M) \oplus \mathscr{H}^{-}(M)$ is not a Hilbert space representation of the $*$-algebra $\mathscr{C}(M)$ but rather a representation on the indefinite space $\left(\mathscr{H}^{+}(M) \oplus \mathscr{H}^{-}(M),\langle\cdot, \cdot\rangle_{L^{2}(M)}\right)$.

Therefore we come up with a natural embedding of classical real Riemannian (or Lorentzian with complexified curvature) vacuum general relativity into a quantum framework:

C. The real Riemannian curvature tensor of an orientable Riemannian Einstein 4-manifold $(M, g)$ is a global section $R_{g} \in \mathscr{C}(M)$ of the sheaf $\mathscr{C}_{M}$. The curvature $R_{g}$ also can be regarded as a linear real self-adjoint operator with respect to the scalar product (2) acting on the canonically split Hilbert space $\mathscr{H}^{+}(M) \oplus \mathscr{H}^{-}(M)$ induced by the metric such that $R_{g}$ obeys this splitting. The existence of a metric breaks the original symmetry group $\operatorname{Diff}^{+}(M)$ down to the finite dimensional group $\operatorname{Iso}^{+}(M, g)$ which acts on $\mathscr{H}^{+}(M) \oplus \mathscr{H}^{-}(M)$ also obeying the splitting.

Remark. Before proceeding further we call attention that - taking into account that under mild technical assumptions both the vacuum $[9,11,14]$ and the non-vacuum [5] Einstein equations admit at least local solutions with prescribed regularity - this classical picture is expected to continue to hold at least locally in the following sense if one considers more general algebraic curvature tensors. Given a connected oriented smooth 4-manifold $M$ with a point $x \in M$ it is known that if a global algebraic curvature tensor $R_{M} \in \mathscr{C}(M)$ satisfies some technical conditions in $x$ (formulated for example in $[9,11,14])$, then there exists at least a local Riemannian Einstein metric $g_{U}$ on an open subset $x \in U \subseteq M$ with the property $\left.R_{g_{U}}\right|_{x}=\left.R_{M}\right|_{x}=R_{x}$ i.e., the two curvature tensors coincide at least in $x$. Apparently we can pick a countable collection of distinguished points of this kind such that the corresponding open subsets comprise an open covering of $M$ hence endowing $M$ with a "patchwork structure" of local Einstein metrics.

2. Next we take a departure from classical general relativity and explore the quantum regime. Of course the trouble is how to describe a generic bounded linear operator $Q \in \mathfrak{B}(M)$ in terms of a geometric linear operator $R \in \mathscr{C}(M) \cap \mathfrak{B}(M)$. Our quantum instinct tells us that a truely quantum operator should be constructed by somehow smearing geometric operators over regions in $M$. This instinct will be justified by the famous Schwartz kernel theorem applied below.

Fix a geometric operator $R \in \mathscr{C}(M) \cap \mathfrak{B}(M)$ and a point $x \in M$. Then on any 2 -form $\omega \in \Omega_{c}^{2}(M ; \mathbb{C})$ its action can be expressed in a fully local form $(R \omega)_{x}=R_{x} \omega_{x}$. We can generalize this as follows. Pick finitely many 
distinct further points $y_{1}, \ldots, y_{n(x)} \in M$ where $n(x) \in \mathbb{N}$ may depend on $x \in M$. Consider diffeomorphisms $f_{y_{i}} \in \operatorname{Diff}^{+}(M)$ such that $f_{y_{0}}=\operatorname{Id}_{U}$ hence $f_{y_{0}}(x)=x$ moreover $f_{y_{i}}(x)=y_{i}$ for $i=1, \ldots, n(x)$. An operator $Q \in \mathfrak{B}(M)$ out of $R \in \mathscr{C}(M) \cap \mathfrak{B}(M)$ and $f_{y_{0}}, \ldots, f_{y_{n(x)}} \in \operatorname{Diff}^{+}(M)$ is constructed such that on vectors $\omega \in \Omega_{c}^{2}(M ; \mathbb{C})$ forming a dense subset has the shape

$$
(Q \omega)_{x}:=\sum_{i=0}^{n(x)} f_{y_{i}}^{*}(R \omega)=R_{x} \omega_{x}+\sum_{i=1}^{n(x)} f_{y_{i}}^{*}(R \omega) .
$$

Note that this linear operator is not local in the sense that its effect on $\omega_{x}$ depends not only on $R_{x}$ and $\omega_{x}$ but on the value of $R$ and $\omega$ in further distant points $y_{1}, \ldots, y_{n(x)} \in M$ as well. The question arises how to generalize this construction for countable or even uncountable infinite sums. For all points $y \in M$ pick up unique diffeomorphisms $f_{y} \in \operatorname{Diff}^{+}(M)$ such that $f_{y}(x)=y$ and $f_{x}=\operatorname{Id}_{M}$. Then for all $\omega \in \Omega_{c}^{2}(M ; \mathbb{C})$ the assignment $y \mapsto f_{y}^{*}(R \omega)$ gives a function from $M$ into $\wedge_{x}^{2} M \otimes_{\mathbb{R}} \mathbb{C}$. Suppose we can integrate it against a complex measure $\mu_{x}$ on $M$ what we write as $\int_{y \in M} f_{y}^{*}(R \omega) \mathrm{d} \mu_{x}(y)$. Such a measure can be constructed from a double 2-form $K$ i.e., a section of the bundle $\left(\wedge^{2} M \otimes_{\mathbb{R}} \mathbb{C}\right) \times\left(\wedge^{2} M \otimes_{\mathbb{R}} \mathbb{C}\right)$ over $M \times M$ regarding it as a "kernel function". In other words for all $x \in M$ and a 2 -form $\omega$ we put

$$
\int_{y \in M} f_{y}^{*}(R \omega) \mathrm{d} \mu_{x}(y):=\int_{y \in M} K_{x, y} \wedge(R \omega)_{y} \in \wedge_{x}^{2} M \otimes_{\mathbb{R}} \mathbb{C} .
$$

Consequently the appropriate way to generalize the discrete formula (6) is to set

$$
(Q \omega)_{x}:=\int_{y \in M} K_{x, y} \wedge(R \omega)_{y}
$$

Of course in order this integral to make sense we have to specialize the precise class of these "kernel functions". We shall not do it here but note that the more singular the kernel is, the more general is the resulting bounded linear operator. The general situation is controlled by the Schwartz kernel theorem: non-tempered distributional double 2-forms $K \in \mathscr{D}^{\prime}\left(M \times M ;\left(\wedge^{2} M \otimes_{\mathbb{R}} \mathbb{C}\right) \times\right.$ $\left.\left(\wedge^{2} M \otimes_{\mathbb{R}} \mathbb{C}\right)\right)$ give rise to bounded linear operators $Q$ via $\langle\alpha, Q \beta\rangle_{L^{2}(M)}=$ $(K, \alpha \otimes(R \beta))_{M \times M}$ where this latter bracket is the pairing between dual spaces (cf. e.g. [19, Vol. I §4.6]) and all bounded linear operators arise this way with suitable kernels.

Q. Over a connected oriented smooth 4-manifold $M$ a generic element $Q \in$ $\mathfrak{B}(M)$ always can be constructed from a geometric one $R \in \mathscr{C}(M) \cap \mathfrak{B}(M)$ 
by a smearing procedure provided by the Schwartz kernel theorem. In this general situation no pointwisely given geometric object has a meaning because the original symmetry group $\operatorname{Diff}^{+}(M)$ is unbroken. This is in accord with the physical expectations.

We have completed the exploration of the elements of $\mathscr{C}(M)$ and $\mathfrak{B}(M)$.

\section{Gravity as an algebraic quantum field theory}

Before proceeding further let us summarize the situation we have reached in Section 2. To a smooth oriented 4-manifold $M$ one can attach a sheaf $\mathscr{C}_{M}$ whose global sections $\mathscr{C}(M)$ contains algebraic curvature tensors. $\mathscr{C}(M)$ often can be completed to a $C^{*}$-algebra $\mathfrak{C}(M)$. Classical solutions of the vacuum Einstein equations i.e., classical real Riemannian (or Lorentzian with complexified curvature) Einstein manifolds $(M, g)$ can be characterized by the fact that their curvature operators obey the canonical splitting $\Omega_{c}^{+}(M ; \mathbb{C}) \oplus \Omega_{c}^{-}(M ; \mathbb{C}) \subset \mathscr{H}^{+}(M) \oplus \mathscr{H}^{-}(M)$ and this completion equipped with an indefinite scalar product carries a representation of $\mathscr{C}(M)$ or even $\mathfrak{C}(M)$ and a unitary one of $\operatorname{Diff}^{+}(M)$. Therefore one is tempted to look at curvature operators as local quantum observables in a quantum field theory possessing a huge symmetry group coming from diffeomorphisms. We make these observations more formal by constructing something which resembles an algebraic quantum field theory in the sense of [10]. For this aim we need a "net" or a "co-presheaf" of local algebras on $M$ i.e., a functorial assignment $O \mapsto \mathfrak{A}(O)$ attaching $C^{*}$-algebras $\mathfrak{A}(O)$ to open subsets $\emptyset \subseteq O \subseteq M$ such that the basic axioms of this theory having still meaning in our more general context should be satisfied.

Recall that the space of local smooth complexified (0,4)-type algebraic curvature tensors over $M$ is $C^{\infty}\left(M ;\left(S^{2} \wedge^{2} M \cap \operatorname{Ker} b\right) \otimes_{\mathbb{R}} \mathbb{C}\right)$ where $b$ : $C^{\infty}\left(M ;\left(\wedge^{1} M\right)^{\otimes 4}\right) \rightarrow C^{\infty}\left(M ;\left(\wedge^{1} M\right)^{\otimes 4}\right)$ is the usual algebraic Bianchi map. Making use of a metric i.e., pseudo-Euclidean structures on the fibers, the corresponding (2,2)-type algebraic curvature tensors fulfill a subspace of $C^{\infty}\left(M ; \operatorname{End}\left(\wedge^{2} M \otimes_{\mathbb{R}} \mathbb{C}\right)\right)$. However now we lack any preferred metric hence only the whole endomorphism space is at our disposal. Consider therefore $\operatorname{End}\left(\Omega_{c}^{2}(M ; \mathbb{C})\right)$, the adjoint operation * with respect to $(1)$ and the norm (4) given by the spectral radius. Take compactly supported complex bundle morphisms $R \in C_{c}^{\infty}\left(M ; \operatorname{End}\left(\wedge^{2} M \otimes_{\mathbb{R}} \mathbb{C}\right)\right)$ and real vector fields $X \in C_{c}^{\infty}(M ; T M)$ with the associated Lie derivative $L_{X}$. Then $\mathrm{e}^{R}$ as well as $\mathrm{e}^{L_{X}}$ have finite norm (4). Fix a relatively compact open subset $\emptyset \subseteq$ 
$U \subseteq M$ and let $\mathfrak{A}(U)$ be the unital $C^{*}$-algebra generated by the operators $\mathrm{e}^{R}, \mathrm{e}^{L_{X}}$ which commute on $\Omega_{c}^{2}(M \backslash U ; \mathbb{C}) \subset \Omega_{c}^{2}(M ; \mathbb{C})$ with the subgroup $\operatorname{Diff}_{U}^{+}(M) \subset \operatorname{Diff}^{+}(M)$ consisting of $U$-preserving diffeomorphisms. I.e., $\mathfrak{A}(U)$ arises as the norm-closure for (4) of the *-closed subspace

$\left\langle\mathrm{e}^{R}, \mathrm{e}^{L_{X}} \mid\left[\left.\mathrm{e}^{R}\right|_{\Omega_{c}^{2}(M \backslash U ; \mathbb{C})}, \operatorname{Diff}_{U}^{+}(M)\right]=0,\left[\left.\mathrm{e}^{L_{X}}\right|_{\Omega_{c}^{2}(M \backslash U ; \mathbb{C})}, \operatorname{Diff}_{U}^{+}(M)\right]=0\right\rangle$.

By construction $\mathbb{C} \cdot 1 \cong \mathfrak{A}(\emptyset) \subseteq \mathfrak{A}(U) \subseteq \mathfrak{A}(V)$ if $\emptyset \subseteq U \subseteq V$ therefore, as usual, the global algebra $\mathfrak{A}(M)$ is constructed (if $M$ is non-compact) as the $C^{*}$-algebra direct (inductive) limit of these local algebras.

Definition 3.1. The algebra $\mathfrak{A}(U)$ is called the local generalized CCR algebra of local quantum observables while $\mathfrak{A}(M)$ is the global generalized CCR algebra of $M$.

Remark. 1. This definition of local quantum observables stems from the physical intuition that on remote localized states local operations should commute with localization-preseving symmetries.

2. $\mathfrak{A}(U)$ contains a usual CCR algebra at least when $U \subseteq M$ is a coordinate ball. Pick self-adjoint local endomorphisms $R$ and local vector fields $X$ with $L_{X}$ being self-adjoint. Since $X$ is real then $\mathrm{e}^{L_{X}}$ is a diffeomorphism which is unitary hence $L_{X}$ is self-adjoint. Consider the maximal subspace of those self-adjoint elements which either commute: $\left[R_{1}, R_{2}\right]=0$, $\left[L_{X_{1}}, L_{X_{2}}\right]=0,\left[R, L_{X}\right]=0$ or are canonically conjugate to each other i.e., $\left[R, L_{X}\right]=c \cdot 1$ with $c \in \mathbb{C}$. Then the sub- $C^{*}$-algebra in $\mathfrak{A}(U)$ generated by the corresponding unitary operators $\mathrm{e}^{R}, \mathrm{e}^{L_{X}}$ form a usual CCR algebra; $R$ and $L_{X}$ play the role of the position operator $\mathbf{Q}$ and its canonically conjugate momentum operator $\mathbf{P}$, respectively. This standard CCR algebra within $\mathfrak{A}(U)$ describes the "free graviton part" while the rest of $\mathfrak{A}(U)$ the "selfinteracting part" of this theory. This justifies in some extent why we expect to construct something like a "quantum theory".

Putting things together then let us consider the algebraic quantum field theory defined by the assignment

$$
U \longmapsto \mathfrak{A}(U), \quad U \subseteq M \text { is relatively compact open. }
$$

Moreover $\mathfrak{A}(M)$ is taken to be the $C^{*}$-algebra direct (inductive) limit of the $\mathfrak{A}(U)$ 's as usual. Note that the formulation of this theory rests only on the smooth structure on $M$ hence does not refer to any metric on $M$ for instance. A Hilbert space $\mathscr{H}^{+}(M) \oplus \mathscr{H}^{-}(M)$ carries an action of all $\mathfrak{A}(U)$ 's from the 
left and a unitary representation with respect to $\langle\cdot, \cdot\rangle_{L^{2}(M)}$ of $\operatorname{Diff}^{+}(M)$ from the right. Elements of the algebra $\mathfrak{A}(U)$ are the local quantum observables and those of the group $\operatorname{Diff}^{+}(M)$ are the symmetry transformations. The states are continuous normalized positive linear functionals on $\mathfrak{A}(M)$ and the expectation value of $B \in \mathfrak{A}(M)$ in the state $\Phi$ is $\Phi(B) \in \mathbb{C}$.

Now we introduce the concept of a "quantum gravitational field" in the standard way.

Definition 3.2. Let $M$ be a connected oriented smooth 4-manifold. Take a local generalized CCR algebra $\mathfrak{A}(U)$ generated by $\mathrm{e}^{R}$ 's and $\mathrm{e}^{L_{X}}$ 's as above. For a differentiable 1-parameter subgroup $\left\{A_{t}\right\}_{t \in \mathbb{R}} \subset \mathfrak{A}(U)$ with $A_{0}=1 \in$ $\mathfrak{A}(U)$ a local observable of the infinitesimal form

$$
Q:=\left.\frac{\mathrm{d} A_{t}}{\mathrm{~d} t}\right|_{t=0} \in T_{1} \mathfrak{A}(U)
$$

is a called a local quantum gravitational field on $U \subseteq M$.

Take any split Hilbert space $\mathscr{H}^{+}(M) \oplus \mathscr{H}^{-}(M)$ containing maximal definite orthogonal subspaces (note that this breaks the diffeomorphism symmetry). The off-blockdiagonal part of $Q$ is the material content of the local quantum gravitational field relative to the splitting. In particular $Q$ is called a local quantum vacuum gravitational field relative to the splitting if its material content relative to the splitting vanishes i.e., $Q\left(\mathscr{H}^{ \pm}(M) \cap D\right) \subseteq$ $\mathscr{H}^{ \pm}(M)$ at least on a dense subset $D \subseteq \mathscr{H}^{+}(M) \oplus \mathscr{H}^{-}(M)$.

Now we turn to the representation theory of the global algebra $\mathfrak{A}(M)$. As usual this global CCR algebra of observables admits an abundance of nonequivalent representations therefore an important task is to single out those which possess some - either mathematical or physical - significance.

Firstly we construct what will be referred to as the tautological representation having probably a mathematical relevance only.

Theorem 3.1. $M$ itself gives rise to a faithful and irreducible so-called tautological representation $\pi_{M}$ of $\mathfrak{A}(M)$ on a Hilbert space $\mathscr{H}_{M}$. It also carries a unitary representation $U_{M}$ of the group $\operatorname{Diff}^{+}(M)$. A vector $v \in$ $\mathscr{H}_{M}$ satisfies $U_{M}(v)=v$ if and only if $v=0$ ("no vacuum").

As a consequence to $M$ always a von Neumann algebra $\mathfrak{R}(M):=$ $\left(\pi_{M}(\mathfrak{A}(M))\right)^{\prime \prime}$ can be attached canonically.

Proof. Referring back to Lemma 2.2 we improve $\mathfrak{A}(M)$ itself to a Hilbert space $\mathscr{H}_{M}$ on which $\mathfrak{A}(M)$ acts from the left. Recall that $\mathfrak{A}(M)$ has a norm 
given by the spectral radius (4). We want to demonstrate that this norm $[[\cdot]]$ actually comes from a positive definite non-degenerate Hermite scalar product $(\cdot, \cdot)_{M}$. This will also yield that the Hilbert space completion $\mathscr{H}_{M}$ of $\mathfrak{A}(M)$ will actually coincide with $\mathfrak{A}(M)$ i.e., $\mathscr{H}_{M}$ will arise simply by putting this scalar product onto $\mathfrak{A}(M)$.

Define a map from $\mathfrak{A}(M)^{\mathbb{R}} \times \mathfrak{A}(M)^{\mathbb{R}}$ into $\mathbb{R}$ by differentiating $T \mapsto[[T]]^{2}$ at the unit $1 \in \mathfrak{A}(M)$ as follows:

$$
\mathfrak{A}(M)^{\mathbb{R}} \times \mathfrak{A}(M)^{\mathbb{R}} \ni(A, B) \longmapsto \frac{1}{4}\left(\mathrm{D}[[\cdot]]^{2}\right)_{1}\left(A^{*} B+B^{*} A\right) \in \mathbb{R} .
$$

Properties of the norm ensure us that this derivative exists and the map is symmetric and $\mathbb{R}$-bilinear. Take any particular Hilbert space $\mathscr{H}^{+}(M) \oplus$ $\mathscr{H}^{-}(M)$ from the proof of Lemma 2.2. Recall the equality $[[A]]=\|A\|$ for all $A \in \mathfrak{A}(M)$ where $\|$. $\|$ is the usual operator norm on this Hilbert space satisfying (3). Then

$\frac{1}{2} \mathrm{D}\left(\frac{\|(\cdot) v\|_{L^{2}(M)}^{2}}{\|v\|_{L^{2}(M)}^{2}}\right)_{1}\left(A^{*} A\right)=\frac{\operatorname{Re}(A * A v, v)_{L^{2}(M)}}{\|v\|_{L^{2}(M)}^{2}}=\frac{\operatorname{Re}(J A v, A J v)_{L^{2}(M)}}{\|v\|_{L^{2}(M)}^{2}}$

hence these derivatives also exist and taking their supremum with respect to $v \in \mathscr{H}^{+}(M) \oplus \mathscr{H}^{-}(M)$ gives $\|A\|^{2}$. Consequently

$$
\begin{aligned}
\frac{1}{2}\left(\mathrm{D}[[\cdot]]^{2}\right)_{1}\left(A^{*} A\right) & =\frac{1}{2} \mathrm{D}\left(\sup _{v \neq 0} \frac{\|(\cdot) v\|_{L^{2}(M)}^{2}}{\|v\|_{L^{2}(M)}^{2}}\right)_{1}\left(A^{*} A\right) \\
& =\sup _{v \neq 0} \frac{1}{2} \mathrm{D}\left(\frac{\|(\cdot) v\|_{L^{2}(M)}^{2}}{\|v\|_{L^{2}(M)}^{2}}\right)_{1}\left(A^{*} A\right)=\|A\|^{2} .
\end{aligned}
$$

This shows that $\frac{1}{2}\left(\mathrm{D}[[\cdot]]^{2}\right)_{1}\left(A^{*} A\right)=\|A\|^{2} \geqq 0$ and equality holds if and only if $A=0$. Therefore $(A, B) \mapsto \frac{1}{4}\left(\mathrm{D}[[\cdot]]^{2}\right)_{1}\left(A^{*} B+B^{*} A\right)$ is a real nondegenerate scalar product on $\mathfrak{A}(M)^{\mathbb{R}}$ with induced norm $[[\cdot]]$. The norm satisfies $[[A]]=[[\mathbf{i} A]]$ over $\mathfrak{A}(M)$ as well therefore putting

$$
\begin{aligned}
(A, B)_{M}:= & \frac{1}{2}\left([[A+B]]^{2}-[[A]]^{2}-[[B]]^{2}\right) \\
& +\frac{\mathbf{i}}{2}\left([[\mathbf{i} A+B]]^{2}-[[\mathbf{i} A]]^{2}-[[B]]^{2}\right)
\end{aligned}
$$

gives rise to a non-degenerate Hermitian scalar product on $\mathfrak{A}(M)$. In other words $\mathfrak{A}(M)$ as a complete normed space has the further structure of a Hilbert space $\mathscr{H}_{M}$ and $\mathfrak{A}(M)$ acts on it(self) from the left yielding a faithful 
irreducible representation $\pi_{M}$ i.e., $\pi_{M}(A) B:=A B$ for all $A \in \mathfrak{A}(M), B \in$ $\mathscr{H}_{M}=\mathfrak{A}(M)$.

Since by construction $\operatorname{Diff}^{+}(M) \subset \mathfrak{A}(M)$ we also obtain a unitary representation $U_{M}(f):=\pi_{M}\left(f^{*}\right)$ and via part (ii) of Lemma 2.1 obviously $v=0$ is the only invariant vector under $U_{M}$ as stated.

Secondly, in a quantum field theory the algebra of quantum observables must possess positive mass and energy representations. Let us therefore construct some representations $\pi_{\Sigma, \omega}$ of our global algebra $\mathfrak{A}(M)$ what we will call positive mass representations. When doing this we touch upon the problem of gravitational mass and energy which is probably the most painful part of current general relativity [18].

Theorem 3.2. Take an oriented closed surface $\Sigma$. Let $\left(\Sigma, p_{1}, \ldots, p_{n}\right)$ denote a generic smooth immersion $i: \Sigma \rightarrow M$ where the points $p_{1}, \ldots, p_{n} \in \Sigma$ are the preimages of the double points of this immersion. Moreover take any closed $\omega \in \Omega_{c}^{2}(M ; \mathbb{C})$. Assume that

(i) $\frac{1}{2 \pi \mathbf{i}} \int_{\Sigma} \omega=1$;

(ii) $\omega$ is non-degenerate along $\Sigma$ and for all complex structures $C=C(\Sigma)$ on $\Sigma$ there exist positive definite unitary holomorphic vector bundle structures on the vector bundle $E:=\left.T M \otimes_{\mathbb{R}} \mathbb{C}\right|_{C}$ over $C \subset M$ compatible with $\omega$ such that $\operatorname{dim}_{\mathbb{C}} H^{0}(C ; \mathscr{O}(E))=4$.

Then $\left(\Sigma, p_{1}, \ldots, p_{n}, \omega\right)$ gives rise to a so-called positive mass representation $\pi_{\Sigma, \omega}$ of $\mathfrak{A}(M)$ on a Hilbert space $\mathscr{H}_{\Sigma, \omega}$ as follows:

(i) $\mathscr{H}_{\Sigma, \omega}$ also carries a unitary representation $U_{\Sigma, \omega}$ of the group $\operatorname{Diff}^{+}(M)$. $A$ vector $v \in \mathscr{H}_{\Sigma, \omega}$ satisfies $U_{\Sigma, \omega}(f) v=v$ for all $f \in \operatorname{Diff}^{+}(M)$ if and only if $v=0$ ("no vacuum");

(ii) On a dense subset of states $0 \neq[A] \in \mathscr{H}_{\Sigma, \omega}$ a complex 4-vector $P_{C, \omega, A} \in$ $H^{0}(C ; \mathscr{O}(E))$ can be defined together with its length $m_{C, \omega, A}:=$ $\left\|P_{C, \omega, A}\right\|_{L^{2}(C)} \geqq 0$ with respect to a natural Hermitian scalar product $(\cdot, \cdot)_{L^{2}(C)}$ on $C^{\infty}(C ; E)$. It has the porperty that if $[1] \in \mathscr{H}_{\Sigma, \omega}$ is a state corresponding to vanishing algebraic curvature $R=0$ then $P_{C, \omega, 1}=0$ hence $m_{C, \omega, 1}=0$.

Proof. (i) A continuous functional $\Phi_{\Sigma, \omega}: \mathfrak{A}(M) \rightarrow \mathbb{C}$ is defined by extending continuously the map

$$
A \longmapsto \Phi_{\Sigma, \omega}(A):=\frac{1}{2 \pi \mathbf{i}} \int_{\Sigma} A \omega \in \mathbb{C}
$$


from $\operatorname{End}\left(\Omega_{c}^{2}(M ; \mathbb{C})\right) \cap \mathfrak{A}(M)$. By assumption (i) $\Phi_{\Sigma, \omega}\left(1^{*} 1\right)=1$ hence $\Phi_{\Sigma, \omega}\left(A^{*} A\right)>0$ if $\|A-1\|<\varepsilon$. Since any $B \in \mathfrak{A}(M)$ can be written as $B=c A$ with $c \in \mathbb{C}$ we see that $\Phi_{\Sigma, \omega}\left(B^{*} B\right)=|c|^{2} \Phi_{\Sigma, \omega}\left(A^{*} A\right) \geqq 0$ consequently $\Phi_{\Sigma, \omega}$ is continuous, normalized and positive. Therefore the GNS construction applies and yields a corresponding representation of the $C^{*}$ algebra $\mathfrak{A}(M)$. Recall that this goes as follows. One has the induced leftmultiplicative Gelfand ideal $I_{\Sigma, \omega}:=\left\{A \in \mathfrak{A}(M) \mid \Phi_{\Sigma, \omega}\left(A^{*} A\right)=0\right\} \subset \mathfrak{A}(M)$. The functional provides us with a well-defined positive definite scalar product $([A],[B])_{\Sigma, \omega}:=\Phi_{\Sigma, \omega}\left(A^{*} B\right)$ on $\mathfrak{A}(M) / I_{\Sigma, \omega}$ with $A \in[A], B \in[B]$ where $[A]:=A+I_{\Sigma, \omega}$, etc. Making use of this scalar product one completes $\mathfrak{A}(M) / I_{\Sigma, \omega}$ to a Hilbert space $\mathscr{H}_{\Sigma, \omega}$ and then lets $\mathfrak{A}(M)$ act from the left by the continuous extension of $\pi_{\Sigma, \omega}(A)[B]:=[A B]$ from $\mathfrak{A}(M) / I_{\Sigma, \omega}$ to $\mathscr{H}_{\Sigma, \omega}$. Since the whole construction is acted upon equivariantly by $\operatorname{Diff}^{+}(M)$ (i.e., all the $M, \mathfrak{A}(M)$ and $\mathscr{H}_{\Sigma, \omega}$ carry induced actions of the diffeomorphism group) two representations $\pi_{\Sigma, \omega}$ and $\pi_{f(\Sigma), f^{*} \omega}$ are considered to be identical and the whole set of these representations will be denoted by $\pi_{\Sigma, \omega}$ with Hilbert space $\mathscr{H}_{\Sigma, \omega}$. In fact two representations $\pi_{\Sigma_{1}, \omega_{1}}$ and $\pi_{\Sigma_{2}, \omega_{2}}$ are unitary equivalent if and only if there is a positive real number $a \in \mathbb{R}^{+}$such that $\Phi_{\Sigma_{2}, \omega_{2}}=a \Phi_{\Sigma_{1}, \omega_{1}}$ hence in particular if exists an element $f \in \operatorname{Diff}^{+}(M)$ satisfying $\Sigma_{2}=f\left(\Sigma_{1}\right)$ and $\omega_{2}=f^{*} \omega_{1}$; consequently our identification is consistent from a representation-theoretic viewpoint as well.

In usual Poincaré-invariant quantum field theory the Hilbert space carries a unitary representation of the space-time symmetry group. Here the "space-time symmetry transformations" are all the diffeomorphisms hence in our algebraic quantum field theory the corresponding infinitesimal transformations are the Lie derivatives with respect to vector fields. We construct a unitary representation $U_{\Sigma, \omega}$ of $\operatorname{Diff}^{+}(M)$ on $\mathscr{H}_{\Sigma, \omega}$ from the left as follows. An element $f \in \operatorname{Diff}^{+}(M)$ arises as limits of products of diffeomorphisms whose infinitesimal generators are compactly supported real vector fields $X \in C_{c}^{\infty}(M ; T M)$. Recalling the construction of $\mathfrak{A}(M)$ we see that if one looks at the pullback $f^{*}$ induced by $f \in \operatorname{Diff}^{+}(M)$ as a linear operator on $\Omega_{c}^{2}(M ; \mathbb{C})$ then $f^{*} \in \mathfrak{A}(M)$ and it is a unitary element. Therefore put $U_{\Sigma, \omega}(f):=\pi_{\Sigma, \omega}\left(f^{*}\right)$ for $f \in \operatorname{Diff}^{+}(M)$. This representation is indeed unitary because

$$
\begin{aligned}
& \left(U_{\Sigma, \omega}(f)[A], U_{\Sigma, \omega}(f)[B]\right)_{\Sigma, \omega} \\
= & \left(\left[f^{*} A\right],\left[f^{*} B\right]\right)_{\Sigma, \omega} \\
= & \Phi_{\Sigma, \omega}\left(\left(f^{*} A\right)^{*}\left(f^{*} B\right)\right) \\
= & \Phi_{\Sigma, \omega}\left(A^{*} B\right)=([A],[B])_{\Sigma, \omega} .
\end{aligned}
$$


The representation $U_{\Sigma, \omega}: \operatorname{Diff}^{+}(M) \rightarrow \mathrm{U}\left(\mathscr{H}_{\Sigma, \omega}\right)$ has a complexified infinitesimal version

$$
\begin{aligned}
u_{\Sigma, \omega}: C_{c}^{\infty}\left(M ; T M \otimes_{\mathbb{R}} \mathbb{C}\right) & \cong \operatorname{Lie}\left(\operatorname{Diff}^{+}(M)\right) \otimes_{\mathbb{R}} \mathbb{C} \\
\longrightarrow \mathfrak{u}\left(\mathscr{H}_{\Sigma, \omega}\right) \otimes_{\mathbb{R}} \mathbb{C} & \cong \operatorname{Lnd}\left(\mathscr{H}_{\Sigma, \omega}\right)
\end{aligned}
$$

whose matrix elements on the dense subspace

$$
D:=\left(\mathfrak{A}(M) \cap \operatorname{End}\left(\Omega_{c}^{2}(M ; \mathbb{C})\right)\right) / I_{\Sigma, \omega} \subset \mathscr{H}_{\Sigma, \omega}
$$

look like

$$
\left([A], u_{\Sigma, \omega}(X)[B]\right)_{\Sigma, \omega}=\lim _{t \rightarrow 0} \Phi_{\Sigma, \omega}\left(A^{*} \frac{\mathrm{e}^{t X} B-B}{t}\right)=\frac{1}{2 \pi \mathbf{i}} \int_{\Sigma} A^{*} L_{X} B \omega .
$$

For real vector fields we have a more geometric description: if $\left\{f_{t}\right\}_{t \in \mathbb{R}} \subset$ $\operatorname{Diff}^{+}(M)$ is a 1-parameter subgroup for $X \in C_{c}^{\infty}(M ; T M)$ then $u_{\Sigma, \omega}(X)[A] \in$ $\mathscr{H}_{\Sigma, \omega}$ satisfies

$$
\lim _{t \rightarrow 0}\left\|u_{\Sigma, \omega}(X)[A]-\left[\frac{f_{t}^{*} A-A}{t}\right]\right\|_{\Sigma, \omega}=0
$$

consequently the $u_{\Sigma, \omega}(X)$ 's are indeed the complexified infinitesimal generators of $\operatorname{Diff}^{+}(M)$ in the representation $U_{\Sigma, \omega}$. It follows from part (i) of Lemma 2.1 that the only invariant vector under $\operatorname{Diff}^{+}(M)$ is $0 \in \mathscr{H}_{\Sigma, \omega}$.

(ii) In usual Poincaré-invariant quantum field theory a 4 dimensional commuting set of infinitesimal space-time symmetries are regarded as infinitesimal translations; the corresponding operators are interpreted as energy-momentum operators acting on the Hilbert space of the theory. However in general one cannot find a distinguished 4 dimensional commuting subspace which could be called as "infinitesimal translations". To overcome this difficulty we will follow Dougan and Mason [7] (or [18, Chapter 8]) to find a distinguished subspace of vector fields by holomorphy.

Consider $E:=\left.T M \otimes_{\mathbb{R}} \mathbb{C}\right|_{\Sigma}$ satisfying $\operatorname{rk}_{\mathbb{C}} E=4$ and $\operatorname{deg} E=0$. The 2form $\omega$ can also be used to construct a Hermitian metric on it. Indeed, a Hermitian form on $M$ is defined by $g(X, Y):=\frac{1}{2}(\omega(\bar{X}, \mathbf{i} Y)-\omega(\overline{\mathbf{i} X}, Y))$ for all $X, Y \in C^{\infty}\left(M ; T M \otimes_{\mathbb{R}} \mathbb{C}\right)$. By assumptions (ii) in the theorem its restriction makes $E$ into a smooth positive definite unitary vector bundle $(E, g)$ over $\Sigma$. Take a connection $\nabla_{E}: C^{\infty}(\Sigma ; E) \rightarrow C^{\infty}\left(\Sigma ; E \otimes_{\mathbb{C}} \wedge^{1} \Sigma\right)$ satisfying $\nabla_{E} g=0$ which means that it is compatible with the unitary structure. Picking any complex structure on $\Sigma$ we can identify it with a compact Riemann surface 
$C=C(\Sigma)$. The $(0,1)$-part $\bar{\partial}_{E}$ of the connection endows $(E, g)$ with the structure of a unitary holomorphic vector bundle over $C$. Its finite dimensional subspace of holomorphic sections is denoted by $H^{0}(C ; \mathscr{O}(E))$. The RiemannRoch-Hirzebruch theorem gives $\operatorname{dim}_{\mathbb{C}} H^{0}(C ; \mathscr{O}(E)) \geqq 4(1-\operatorname{genus}(C))$ but by assumptions (ii) in the theorem this vector space is supposed to be precisely 4 dimensional. It also follows that the Hermitian $L^{2}$ scalar product $(X, Y)_{L^{2}(C)}:=\frac{1}{2 \pi \mathbf{i}} \int_{C} g(X, Y) \omega$ on $C^{\infty}(C ; E)$ is positive definite. A simple choice for $E$ can be the holomorphically trivial bundle $C \times \mathbb{C}^{4}$.

We already have seen that the expectation value of any vector field $X$ on $M$ is well defined for a dense subset of vectors $0 \neq v \in D \subset \mathscr{H}_{\Sigma, \omega}$ and looks like $\frac{\left(v, u_{\Sigma, \omega}(X) v\right)_{\Sigma, \omega}}{\|v\|_{\Sigma, \omega}^{2}} \in \mathbb{C}$. However

$$
\left([A], u_{\Sigma, \omega}(X)[A]\right)_{\Sigma, \omega}=\frac{1}{2 \pi \mathbf{i}} \int_{\Sigma} A^{*} L_{X} A \omega
$$

by construction, therefore

$$
\frac{\left([A], u_{\Sigma, \omega}(X)[A]\right)_{\Sigma, \omega}}{\|[A]\|_{\Sigma, \omega}^{2}}=\frac{\int_{\Sigma} A^{*} L_{X} A \omega}{\int_{\Sigma} A^{*} A \omega}
$$

which is complex linear in $X \in C_{c}^{\infty}\left(M ; T M \otimes_{\mathbb{R}} \mathbb{C}\right)$. Let $N_{\varepsilon}(C) \subset M$ be a small tubular neighbourhood of $C \subset M$; we can suppose that it is a $B_{\varepsilon}^{2}$ bundle over $C$ hence put $N_{0}(C):=C$. Take a linear functional on $C^{\infty}\left(N_{\varepsilon}(C) ; T N_{\varepsilon}(C) \otimes_{\mathbb{R}} \mathbb{C}\right)$ by setting

$$
P_{\varepsilon, C, \omega, A}\left(\left.X\right|_{N_{\varepsilon}(C)}\right):=\frac{\int_{C} A^{*} L_{X} A \omega}{\int_{C} A * A \omega}
$$

and then on $C^{\infty}(C ; E)$ by

$$
P_{C, \omega, A}\left(\left.X\right|_{C}\right):=\lim _{\varepsilon \rightarrow 0} P_{\varepsilon, C, \omega, A}\left(\left.X\right|_{N_{\varepsilon}(C)}\right)
$$

A vector field $X \in C_{c}^{\infty}\left(M ; T M \otimes_{\mathbb{R}} \mathbb{C}\right)$ is called a quasilocal infinitesimal translation along $\Sigma$ if $\left.\left.X\right|_{C} \in H^{0}(C ; \mathscr{O}(E)) \subset C^{\infty}(C ; E)\right)$ and (7) gives rise to a well-defined dual vector $P_{C, \omega, A} \in\left(H^{0}(C ; \mathscr{O}(E))\right)^{*}$. This $P_{C, \omega, A}$ is called the quasilocal energy-momentum 4-vector along $\Sigma$ of the state $[A] \in \mathscr{H}_{\Sigma, \omega}$. By the aid of the scalar product $(\cdot, \cdot)_{L^{2}(C)}$ we identify $\left(H^{0}(C ; \mathscr{O}(E))\right)^{*}$ with $\overline{H^{0}(C ; \mathscr{O}(E))}$ therefore we can suppose that $P_{C, \omega, A} \in H^{0}(C ; \mathscr{O}(E))$ by 
putting $\left(\bar{P}_{C, \omega, A},\left.X\right|_{C}\right)_{L^{2}(C)}:=P_{C, \omega, A}\left(\left.X\right|_{C}\right)$. By assumptions (ii) of the theorem $P_{C, \omega, A}$ is indeed a complex 4 -vector. Its length looks like

$$
\begin{aligned}
\left\|P_{C, \omega, A}\right\|_{L^{2}(C)}^{2} & =\frac{1}{2 \pi \mathbf{i}} \int_{C} g\left(P_{C, \omega, A}, P_{C, \omega, A}\right) \omega \\
& =\frac{1}{2 \pi \mathbf{i}} \int_{C} \bar{P}_{C, \omega, A} \wedge *_{g} P_{C, \omega, A}=\frac{1}{2 \pi \mathbf{i}} \int_{C}\left|P_{C, \omega, A}\right|_{g}^{2} \omega
\end{aligned}
$$

and we call the number $m_{C, \omega, A}:=\left\|P_{C, \omega, A}\right\|_{L^{2}(C)} \geqq 0$ the mass of the the state $[A] \in \mathscr{H}_{\Sigma, \omega}$.

Finally, the "semiclassical gravitational vacuum" defined by $R=0$ along $M$ is represented by the state $\left[\mathrm{e}^{0}\right] \in \mathscr{H}_{\Sigma, \omega}$. However $\mathrm{e}^{0}=1 \in \mathfrak{A}(M)$ hence $\left[\mathrm{e}^{0}\right]=[1]$. Consequently with some $\varepsilon>0$ for any quasilocal infinitesimal translation $X$ along $\Sigma$ we find

$$
P_{\varepsilon, C, \omega, 1}\left(\left.X\right|_{N_{\varepsilon}(C)}\right)=\frac{1}{2 \pi \mathbf{i}} \int_{C} L_{X} \omega=\frac{1}{2 \pi \mathbf{i}} \int_{C}\left(\iota_{X} \mathrm{~d} \omega+\mathrm{d}\left(\iota_{X} \omega\right)\right)=0
$$

because both $C$ and $\omega$ are closed by assumption. Therefore taking $\varepsilon \rightarrow 0$ the expression (7) yields $P_{C, \omega, 1}=0$ that is, this state has zero quasilocal energy-momentum hence mass as expected.

Remark. 1. The formula (7) for the quasilocal energy-momentum formally remains meaningful for quantum gravitational fields introduced in Definition 3.2. Hence the corresponding quantities $P_{C, \omega, Q}$ and $m_{C, \omega, Q}$ are interpreted as the quasilolcal energy-momentum 4-vector and the mass of a quantum gravitational field $Q$. Among local quantum gravitational fields one can recognize classical curvature tensors hence we obtain quasilocal quantities for classical general relativity, too.

2. Notice that the topological condition for the existence of a representation $\pi_{\Sigma, \omega}$ is that both $i: \Sigma \uparrow M$ and $\omega \in \Omega_{c}^{2}(M ; \mathbb{C})$ must represent nontrivial classes in $H_{2}(M ; \mathbb{Z})$ and $H^{2}(M ; \mathbb{C})$ respectively such that $\langle[\Sigma],[\omega]\rangle_{M}=$ $\frac{1}{2 \pi \mathbf{i}} \int_{\Sigma} \omega \neq 0$. Hence in particular $\mathbb{R}^{4}$ or $S^{4}$ does not possess positive mass representations! However even if $\left[\Sigma_{1}\right]=\left[\Sigma_{2}\right] \in H_{2}(M ; \mathbb{Z})$ and $\left[\omega_{1}\right]=\left[\omega_{2}\right] \in$ $H^{2}(M ; \mathbb{C})$ the resulting representations $\pi_{\Sigma_{1}, \omega_{1}}$ and $\pi_{\Sigma_{2}, \omega_{2}}$ are not unitarily equivalent in general.

Thirdly, apart from the tautological and positive mass quantum representations with unbroken symmetry $\operatorname{Diff}^{+}(M)$ there exist other ones what we call classical representations because in these representations the original vast 
symmetry group is spontaneously broken to a finite dimensional subgroup $\mathrm{Iso}^{+}(M, g) \subset \operatorname{Diff}^{+}(M)$ of an emergent metric $g$ on $M$.

Theorem 3.3. Take a perhaps non-compactly supported $\omega \in \Omega^{2}(M ; \mathbb{C})$ such that $\omega$ is non-degenerate along the whole $M$ moreover satisfies $\int_{M} \bar{\omega} \wedge \omega=1$.

Then $\omega$ gives rise to a so-called classical representation $\pi_{\omega}$ of $\mathfrak{A}(M)$ on a Hilbert space $\mathscr{H}_{\omega}$ as follows:

(i) $\mathscr{H}_{\omega}$ also carries a unitary representation $U_{\omega}$ of the group $1 \subseteq$ $\mathrm{Iso}^{+}(M, g) \varsubsetneqq \operatorname{Diff}^{+}(M)$ consisting of the isometries of the unitary metric $g$ on the complexified tangent bundle given by

$$
g(X, Y):=\frac{1}{2}(\omega(\bar{X}, \mathbf{i} Y)-\omega(\overline{\mathbf{i} X}, Y)) \quad \text { for all } X, Y \in C^{\infty}\left(M ; T M \otimes_{\mathbb{R}} \mathbb{C}\right) .
$$

Moreover the state $\Omega:=[1] \in \mathscr{H}_{\omega}$ corresponding to vanishing algebraic curvature $R=0$ satisfies $U_{\omega}(f) \Omega=\Omega$ for all $f \in \mathrm{Iso}^{+}(M, g)$;

(ii) The distinguished splitting $\mathscr{H}^{+}(M) \oplus \mathscr{H}^{-}(M)$ via (anti)self-duality with respect to $g$ induces a splitting $\mathscr{H}_{\omega}=\mathscr{H}_{\omega}^{+} \oplus \mathscr{H}_{\omega}^{-}$into orthogonal subspaces obeyed by $\mathrm{Iso}^{+}(M, g)$. The distinguished quantum gravitational field $Q:=R_{g}$ in the sense of Definition 3.2 provided by the curvature of the metric $g$ acts on $\mathscr{H}_{\omega}$. Moreover $\pi_{\omega}\left(R_{g}\right)$ obeys the splitting of $\mathscr{H}_{\omega}$ if and only if $R_{g}$ does the same on $\mathscr{H}^{+}(M) \oplus \mathscr{H}^{-}(M)$ i.e., $R_{g}$ is a vacuum quantum gravitational field or in other words $g$ is a complexified Einstein metric on $M$. In particular if the metric $g$ is flat then $R_{g}=0$ also gives the invariant state $\Omega=[1] \in \mathscr{H}_{\omega}$.

Proof. (i) This time take another natural normalized linear functional $\Psi_{\omega}$ : $\mathfrak{A}(M) \rightarrow \mathbb{C}$ by continuously extending a functional whose shape on elements $A \in \operatorname{End}\left(\Omega_{c}^{2}(M ; \mathbb{C})\right) \cap \mathfrak{A}(M)$ looks like

$$
A \longmapsto \Psi_{\omega}(A):=\int_{M} \bar{\omega} \wedge(A \omega)=\langle\omega, A \omega\rangle_{L^{2}(M)}
$$

provided by (1). Exactly as in the proof of Theorem 3.2 we can exploit the continuity of the functional to conclude from $\Psi_{\omega}(1 * 1)=1$ that $\Psi_{\omega}$ is a positive functional on $\mathfrak{A}(M)$.

Therefore applying again the GNS construction we come up with a reprsentation $\pi_{\omega}$ on a Hilbert space $\mathscr{H}_{\omega}$. The metric also provides us with its isometry group $1 \subseteq \mathrm{Iso}^{+}(M, g) \subset \operatorname{Diff}^{+}(M)$. We construct a unitary representation $U_{\omega}$ of $\operatorname{Iso}^{+}(M, g)$ on $\mathscr{H}_{\omega}$ as follows. First of all for any $f \in$ 
Iso ${ }^{+}(M, g)$ we find $f^{*} \mathfrak{A}(M)\left(f^{-1}\right)^{*} \subseteq \mathfrak{A}(M)$. We define a representation on $\mathscr{H}_{\omega}$ by $U_{\omega}(f)[A]:=\left[f^{*} A\left(f^{-1}\right)^{*}\right]$. Moreover diffeomorphisms are unitary: $\left(f^{*}\right)^{*}=\left(f^{-1}\right)^{*}$ and in particular an isometry has the property $\omega=f^{*} \omega$ consequently

$$
\begin{aligned}
& \left(U_{\omega}(f)[A], U_{\omega}(f)[B]\right)_{\omega} \\
= & \int_{M} \bar{\omega} \wedge\left(\left(f^{*} A\left(f^{-1}\right)^{*}\right)^{*}\left(f^{*} B\left(f^{-1}\right)^{*} \omega\right)\right)=\int_{M} \bar{\omega} \wedge\left(f^{*} A^{*} B\left(f^{-1}\right)^{*} \omega\right) \\
= & \int_{M} \overline{f^{*} \omega} \wedge\left(f^{*} A^{*} B \omega\right)=\int_{M} f^{*}\left(\bar{\omega} \wedge\left(A^{*} B \omega\right)\right)=\int_{M} \bar{\omega} \wedge\left(A^{*} B \omega\right) \\
= & ([A],[B])_{\omega}
\end{aligned}
$$

ensuring us that this representation is indeed unitary. $\Omega:=[1] \in \mathscr{H}_{\omega}$ corresponding to the "semiclassical gravitational vacuum" $R=0$ is a (not necessarily unique) invariant vector.

(ii) Since $\mathfrak{A}(M) \subset \operatorname{End}\left(\mathscr{H}^{+}(M) \oplus \mathscr{H}^{-}(M)\right)$ we get a decomposition of $\mathfrak{A}(M)$ as

$$
\begin{aligned}
\mathfrak{A}(M) \cap & \left(\operatorname{End}\left(\mathscr{H}^{+}(M)\right) \oplus \operatorname{End}\left(\mathscr{H}^{-}(M)\right)\right. \\
& \left.\oplus \operatorname{Hom}\left(\mathscr{H}^{+}(M), \mathscr{H}^{-}(M)\right) \oplus \operatorname{Hom}\left(\mathscr{H}^{-}(M), \mathscr{H}^{+}(M)\right)\right) .
\end{aligned}
$$

Write an element $B \in \operatorname{End}\left(\Omega_{c}^{2}(M ; \mathbb{C})\right) \cap \mathfrak{A}(M)$ in the corresponding form as $B=\left(\begin{array}{ll}a & b \\ c & d\end{array}\right)$. It is easy to check that $\omega$ hence $\bar{\omega}$ is (anti)self-dual with respect to $g$ and the orientation on $M$ (on a complex manifold with its natural orientation $\omega$ is always self-dual, cf. [6, Lemma 2.1.57]). Suppose now that $*_{g} \omega=\omega$. Then we obtain $B \omega=a \omega+c \omega$ with $a \omega \in \mathscr{H}^{+}(M)$ as well as $c \omega \in \mathscr{H}^{-}(M)$. Consequently exploiting the orthogonality of $\mathscr{H}^{+}(M)$ and $\mathscr{H}^{-}(M)$ we can expand $\Psi_{\omega}\left(B^{*} B\right)$ and find

$$
\Psi_{\omega}\left(\left(\begin{array}{ll}
a^{*} a+c^{*} c & a^{*} b+c^{*} d \\
b^{*} a+d^{*} c & b^{*} b+d^{*} d
\end{array}\right)\right)=\Psi_{\omega}\left(\left(\begin{array}{cc}
a^{*} a+c^{*} c & 0 \\
0 & 0
\end{array}\right)\right)
$$

yielding that $\mathfrak{A}(M) \cap\left(\operatorname{Hom}\left(\mathscr{H}^{-}(M), \mathscr{H}^{+}(M)\right) \oplus \operatorname{End}\left(\mathscr{H}^{-}(M)\right) \subseteq I_{\omega}\right.$ where, as before, $I_{\omega} \subset \mathfrak{A}(M)$ is the Gelfand ideal of $\Psi_{\omega}$. Consequently $\mathscr{H}_{\omega}-$ being the completion of $\mathfrak{A}(M) / I_{\omega}$ with respect to the scalar product $(\cdot, \cdot)_{\omega}$ — splits like $\mathscr{H}_{\omega}^{+} \oplus \mathscr{H}_{\omega}^{-}$by completing $\left(\mathfrak{A}(M) \cap \operatorname{End}\left(\mathscr{H}^{+}(M)\right)\right) / I_{\omega}$ and $\left(\mathfrak{A}(M) \cap \operatorname{Hom}\left(\mathscr{H}^{+}(M), \mathscr{H}^{-}(M)\right)\right) / I_{\omega}$ respectively. The two summands are orthogonal subspaces and the decomposition obviously satisfies $U_{\omega}\left(\mathscr{H}_{\omega}^{ \pm}\right) \subseteq$ $\mathscr{H}_{\omega}^{ \pm}$. The case of $*_{g} \omega=-\omega$ is similar. 
If $Q:=R_{g}$ is the curvature of $g$ regarded as a quantum gravitational field as in Definition 3.2 and $g$ is vacuum i.e., Einstein then we already know that $R_{g}\left(\mathscr{H}^{ \pm}(M)\right) \subseteq \mathscr{H}^{ \pm}(M)$. Moreover $R_{g} \in T_{1} \mathfrak{A}(M)$ acts on $\mathscr{H}_{\omega}$ from the left by passing to the infinitesimal action of $\mathfrak{A}(M)$ on $\mathscr{H}_{\omega}$ what we continue to denote by $\pi_{\omega}$. It then follows from $\left(\begin{array}{ll}p & 0 \\ 0 & q\end{array}\right)\left(\begin{array}{ll}a & 0 \\ c & 0\end{array}\right)=\left(\begin{array}{ll}p a & 0 \\ q c & 0\end{array}\right)$ that for an Einstein metric $\pi_{\omega}\left(R_{g}\right)$ also satisfies $\pi_{\omega}\left(R_{g}\right)\left(\mathscr{H}_{\omega}^{ \pm}\right) \subseteq \mathscr{H}_{\omega}^{ \pm}$. The particular case of the flat metric with $R_{g}=0$ gives the invariant state $\Omega=[1] \in \mathscr{H}_{\omega}$ as well.

Remark. The usual axioms of algebraic quantum field theory (cf. e.g. [10, pp. 58-60 or pp. 105-107]) typically make no sense in this very general setting. But for clarity we check them one-by-one in order to see in what extent our algebraic quantum field theory is more general than the usual ones. $^{3}$

[10, Axiom A on p. 106] can be translated to saying that the Hilbert space of a representation of the global generalized CCR algebra $\mathfrak{A}(M)$ also carries a unitary representation of the (spontaneously broken) space-time symmetry group of the theory which has been taken to be the whole diffeomorphism group here. We found three types of representations; here we discuss two of them.

We constructed $\mathscr{H}_{\Sigma, \omega}$ carrying a positive mass representation $\pi_{\Sigma, \omega}$ of $\mathfrak{A}(M)$ as well as a unitary representation $U_{\Sigma, \omega}$ of the unbroken group $\operatorname{Diff}^{+}(M)$. However $\mathscr{H}_{\Sigma, \omega}$ does not possess a $\operatorname{Diff}^{+}(M)$-invariant state i.e., "vacuum" does not exist here. Nevertheless the Dougan-Mason quasilocal translations of $i: \Sigma \leftrightarrow \rightarrow M$ give rise to quasilocal energy-momentum 4-vectors $P_{C, \omega, A}$ in a manner that the state corresponding to the classical gravitational vacuum has vanishing energy-momentum as one expects. This is interesting because the concepts of mass and energy are quite problematic in classical general relativity as well as that of the vacuum in general quantum field theories. But recall that this construction - which mixes ideas of quasilocal constructions in classical general relativity $[7,18]$ and standard GNS representation theory of $C^{*}$-algebras - contains a technical ambiguity namely a

\footnotetext{
${ }^{3}$ We quote from Haag [10, p. 60]: "On the other hand the word $\gg$ axiom $\ll$ suggests something fixed, unchangeable. This is certainly not intended here. Indeed, some of the assumptions are rather technical and should be replaced by some more natural ones as deeper insight is gained. We are concerned with a developing area of physics which is far from closed and should keep an open mind for modifications of assumptions, additional structural principles as well as information singling out a specific theory within the general frame."
} 
choice of a complex structure on an immersed surface in $M$. However one expects the whole machinery to be independent of this choice. We treat this problem in Section 4.

We also constructed $\mathscr{H}_{\omega}$ carrying a classical representation $\pi_{\omega}$ of $\mathfrak{A}(M)$ together with a unitary representation $U_{\omega}$ of the spontaneously broken group $\mathrm{Iso}^{+}(M, g) \subset \operatorname{Diff}^{+}(M)$ provided by an emergent metric $g$ on $M$. This representation gives back the classical picture. It also possesses a (probably not unique) invariant state $\Omega \in \mathscr{H}_{\omega}$ but this time we lack the concept of energy-momentum hence we cannot call this state as the "vacuum".

[10, Axioms $\mathbf{B}$ and $\mathbf{C}$ on p. 107] dealing with the additivity of local algebras and their hermiticity by construction hold here.

[10, Axiom $\mathbf{D}$ on p. 107] can be translated to saying that since the diffeomorphism group is the symmetry group of the theory, it acts on the net of local algebras like

$$
f^{*} \mathfrak{A}(U)\left(f^{-1}\right)^{*}=\mathfrak{A}(f(U))
$$

for all $f \in \operatorname{Diff}^{+}(M)$ i.e., symmetry transformations map the local algebra of a region to that one of the transformed region. This continues to be valid here.

[10, Axiom $\mathbf{E}$ on p. 107] holds in a trivial way as an unavoidable consequence of the vast diffeomorphism invariance. It is easy to see that $[\mathfrak{A}(U), \mathfrak{A}(V)]=0$ if and only if $U \cap V=\emptyset$. Indeed, demanding (8) to be valid we can see that regardless what $\mathfrak{A}(U)$ actually is, it must commute with diffeomorphisms being the identity on $U$; consequently if $A \in \mathfrak{A}(U) \subset$ $\mathfrak{B}(M)$ then $\left.A\right|_{\Omega_{c}^{2}(M \backslash U ; \mathbb{C})} \in \mathfrak{Z}(\mathfrak{B}(M \backslash U))=\mathbb{C} \operatorname{Id}_{\Omega_{c}^{2}(M \backslash U ; \mathbb{C})}$. But $\Omega_{c}^{2}(V ; \mathbb{C}) \subset$ $\Omega_{c}^{2}(M \backslash U ; \mathbb{C})$ if $U \cap V=\emptyset$ hence the assertion follows. Therefore there is no causality hence no dynamics present here. Hence the reason we prefer to use Riemannian metrics over Lorentzian ones throughout the paper (although emphasize again that all conclusions hold for Lorentzian metrics as well). We can also physically say that this theory represents a very elementary level of physical reality where even no causality exists yet. Causality should emerge through breaking of the diffeomorphism symmetry. This symmetry breaking has been carried out in the case of the classical representations.

[10, Axiom $\mathbf{F}$ on p. 107] This completeness requirement claims for the validity of Schur's lemma i.e., in a representation the only bounded operator which commutes with all quantum observables should be a multiple of the identity operator. This holds if the representation of $\mathfrak{A}(M)$ in question is irreducible. 
[10, Axiom G on p. 107] about "primitive causality" has no meaning in this general setting.

\section{Positive mass representations and conformal field theory}

Theorem 3.2 allows us to make a link with conformal field theory. We obtained representations $\pi_{\Sigma, \omega}$ of the algebra of global observables $\mathfrak{A}(M)$ constructed by standard means from a smooth immersion $\left(\Sigma, p_{1}, \ldots, p_{n}\right)$ of a surface $\Sigma$ into $M$ and a regular element $\omega \in \Omega_{c}^{2}(M ; \mathbb{C})$. If a complex structure $C=C(\Sigma)$ is put onto the surface as well then the quasilocal energymomentum $P_{C, \omega, A} \in H^{0}(C ; \mathscr{O}(E))$ and mass $m_{C, \omega, A} \in \mathbb{R}^{+} \cup\{0\}$ of a nonzero state $[A] \in \mathscr{H}_{\Sigma, \omega}$ can be defined enriching $\pi_{\Sigma, \omega}$ further to a positive mass representation. However on physical grounds we expect the whole construction to be independent of these technicalities i.e., any choice of these complex structures have to result in the same construction. Following Witten [21] this means that a conformal field theory lurks behind the curtain. We can indeed find this theory which however turns out to be a very simple topological conformal field theory in the sense that its Hilbert space is finite dimensional and the correlation functions are insensitive for the insertion of marked points i.e., how the immersion looks like.

In constructing this topological conformal field theory we will follow G. Segal [16]. That is first construct a "modular functor extended with an Abelian category possessing a symmetric object" (cf. [1, Definition 5.1.12]) in particular and [1, Chapters 5 and 6$]$ in general). In other words we have to construct an assignment

$$
\tau:\left(\Sigma, p_{1}, \ldots, p_{n}\right) \longmapsto \tau\left(\Sigma, p_{1}, \ldots, p_{n}\right)
$$

which somehow associates to surfaces with marked points finite dimensional complex vector spaces satisfying certain axioms. Consider a positive mass representation $\pi_{\Sigma, \omega}$ of $\mathfrak{A}(M)$ constructed out of $\left(\Sigma, p_{1}, \ldots, p_{n}, \omega\right)$ as in Theorem 3.2. Recall that the marked points $p_{i} \in \Sigma$ correspond the multiple points of the immersion $i: \Sigma \uparrow M$ (the case $(\Sigma, \emptyset, \omega)$ is an embedding). Then to a positive mass representation of $\mathfrak{A}(M)$ a holomorphic vector bundle $\mathscr{E}$ of spaces of conformal blocks $\tau\left(\Sigma, p_{1}, \ldots, p_{n}\right)$ over the coarse moduli space $\mathscr{M}_{g, n}$ of complex structures on $\left(\Sigma, p_{1}, \ldots, p_{n}\right)$ will be assigned in manner that if $0 \neq[A] \in \mathscr{H}_{\Sigma, \omega}$ is a state then its quasilocal energy-momentum 4-vector $P_{C, \omega, A}$ gives rise to a section $P_{\Sigma, \omega, A}$ of $\mathscr{E}$. This section will be moreover (projectively) flat with respect to the natural (projectively) flat connection $\nabla$ on $\mathscr{E}$ (the Knizhnik-Zamolodchikov connection). In other words 
the quasilocal energy-momentum 4-vector gives rise to a conformal block in this conformal field theory.

We begin with the following simple observation (an elementary version of Uhlenbeck's singularity removal theorem [20]).

Lemma 4.1. Take any compact Riemann surface $C=C(\Sigma)$ with distinct marked points $p_{1}, \ldots, p_{n} \in C$ and a holomorphic unitary vector bundle $F^{\prime}$ over $C \backslash\left\{p_{1}, \ldots, p_{n}\right\}$. Let $s^{\prime} \in H^{0}\left(C \backslash\left\{p_{1}, \ldots, p_{n}\right\} ; \mathscr{O}\left(F^{\prime}\right)\right)$ be a meromorphic section with the property $\left\|s^{\prime}\right\|_{L_{l o c}^{2}(C)}<+\infty$ i.e., having locally finite energy over $C$.

If $s^{\prime}$ is singular in $p_{i} \in C$ then one can find a local gauge transformation about this point such that the gauge transformed section extends holomorphically across it i.e., pointlike singularities of locally finite energy meromorphic sections over $C$ are removable. More precisely there exists a unique unitary holomorphic vector bundle $F$ over $C$ satisfying $\left.F\right|_{C \backslash\left\{p_{1}, \ldots, p_{n}\right\}} \cong F^{\prime}$ so that for any locally finite energy section $s^{\prime} \in H^{0}\left(C \backslash\left\{p_{1}, \ldots, p_{n}\right\} ; \mathscr{O}\left(F^{\prime}\right)\right)$ there exists a section $s \in H^{0}(C ; \mathscr{O}(F))$ with the property $\left.s\right|_{C \backslash\left\{p_{1}, \ldots, p_{n}\right\}}$ is gauge equivalent to $s^{\prime}$.

Proof. First we prove the existence of the unique extendibility of the unitary bundle $\left(F^{\prime}, h^{\prime}\right)$. Consider a local holomorphic coordinate system $\left(U_{i}, z\right)$ on $C$ such that $z\left(U_{i}\right)=D(0) \subset \mathbb{C}$ some open disc about the origin and $U_{i}$ contains only one marked point $p_{i} \in U_{i}$ satisfying $z\left(p_{i}\right)=0$. Cutting out the open neighbourhood $U_{i} \subset C$ of $p_{i}$ we obtain a manifold-with-boundary $C \backslash U_{i}$ and $\partial\left(C \backslash U_{i}\right) \cong S^{1}$. Consider the restriction $\left.\left(F^{\prime}, h^{\prime}\right)\right|_{\partial\left(C \backslash U_{i}\right)}$ regarded as a smooth $\mathrm{U}(k)$-bundle over $S^{1}$. Taking a smooth local trivialization the corresponding smooth local transition function of $\left.\left(F^{\prime}, h^{\prime}\right)\right|_{\partial\left(C \backslash U_{i}\right)}$ gives rise to a monodromy map $\mu_{i}: S^{1} \rightarrow \mathrm{U}(k)$ where $k=\operatorname{rk} F^{\prime}$. However $\pi_{0}(\mathrm{U}(k)) \cong 1$ hence this monodromy map together with its derivatives along $S^{1}$ extends over $p_{i}$ as the identity consequently $\left.\left(F^{\prime}, h^{\prime}\right)\right|_{U_{i} \backslash\left\{p_{i}\right\}}$ can be extended over this point as a smooth unitary vector bundle $\left.\left(F_{i}, h_{i}\right)\right|_{U_{i}}$. Consider a smooth trivialization $\left.F_{i}\right|_{U_{i}} \cong U_{i} \times \mathbb{C}^{k}$ and write in this smooth gauge the restriction of the partial connection defining the holomorphic structure on $F^{\prime}$ as $\left.\bar{\partial}_{F^{\prime}}\right|_{U_{i} \backslash\left\{p_{i}\right\}}=\bar{\partial}+\alpha_{U_{i} \backslash\left\{p_{i}\right\}}^{\prime}$. Then the Hermitian scalar product on $F_{i}$ satisfies

$$
\bar{\partial}_{F_{i}}\left(\left.h_{i}\right|_{U_{i} \backslash\left\{p_{i}\right\}}\right)=\bar{\partial}\left(\left.h_{i}\right|_{U_{i} \backslash\left\{p_{i}\right\}}\right)+\alpha_{U_{i} \backslash\left\{p_{i}\right\}}^{\prime}\left(\left.h_{i}\right|_{U_{i} \backslash\left\{p_{i}\right\}}\right)=0
$$

and $\left.h_{i}\right|_{U_{i} \backslash\left\{p_{i}\right\}}$ extends smoothly over $p_{i}$ as $\left.h_{i}\right|_{U_{i}}$. Therefore $\alpha_{U_{i}}:=$ - $\left(\left.\bar{\partial} h_{i}\right|_{U_{i}}\right)\left(\left.h_{i}\right|_{U_{i}}\right)^{-1}$ on $U_{i}$ defines a smooth extension of $\alpha_{U_{i} \backslash\left\{p_{i}\right\}}^{\prime}$ over $p_{i}$ in a manner that $\left.\bar{\partial}_{F_{i}}\right|_{U_{i}}:=\bar{\partial}+\alpha_{U_{i}}$ is the restriction of a compatible partial 
connection $\bar{\partial}_{F_{i}}$ yielding a compatible holomorphic structure on $\left(F_{i}, h_{i}\right)$. Performing this procedure around every marked points we obtain a unique unitary holomorphic vector bundle i.e., $\left(F, h, \bar{\partial}_{F}\right)$ with $\bar{\partial}_{F} h=0$.

Now we come to the extendibility of sections. Compatibility provides us that in a local holomorphic trivialization $\left.F\right|_{U} \cong U \times \mathbb{C}^{k}$ the coefficients of $\left.h\right|_{U}$ are holomorphic functions. Performing a $\mathrm{GL}(k, \mathbb{C})$-valued holomorphic gauge transformation if necessary we can pass to a local holomorphic trivialization in which $\left.h\right|_{U}$ has the standard form. Take any holomorphic section of $F$ or equivalently, a meromorphic section of $F$ with singularities in the marked points i.e., pick any

$$
s^{\prime} \in H^{0}\left(C \backslash\left\{p_{1}, \ldots, p_{n}\right\} ; \mathscr{O}\left(F^{\prime}\right)\right) \cong H^{0}\left(C \backslash\left\{p_{1}, \ldots, p_{n}\right\} ; \mathscr{O}(F)\right)
$$

with local shape $\left.s^{\prime}\right|_{U}(z)=s^{1}(z) f_{1}+\cdots+s^{k}(z) f_{k}$ in this local trivialization. Since $\left.s^{\prime}\right|_{U}$ is holomorphic outside $0 \in \mathbb{C}$ each components $s^{\prime j}: U \rightarrow \mathbb{C}$ admit Laurent expansions

$$
s^{\prime j}(z)=\sum_{m=-N^{j}}^{+\infty} a_{m}^{j} z^{m}, \quad a_{m}^{j} \in \mathbb{C} .
$$

Moreover the local $L^{2}$-norm of the section in this special gauge looks like

$$
\begin{aligned}
\left\|s^{\prime}\right\|_{L^{2}(U)}^{2} & =\left.\frac{1}{2 \pi \mathbf{i}} \int_{U}\left(\left|s^{\prime 1}(z)\right|^{2}+\cdots+\left|s^{\prime k}(z)\right|^{2}\right) \omega\right|_{U} \\
& =\int_{U}\left(\left|s^{\prime 1}(z)\right|^{2}+\cdots+\left|s^{\prime k}(z)\right|^{2}\right) \varphi_{U}(z, \bar{z}) \mathrm{d} z \wedge \mathrm{d} \bar{z}
\end{aligned}
$$

where $\varphi_{U}$ is a smooth nowhere vanishing function on $U$. Assume that the section has locally finite energy. On substituting the above expansions into this integral the finiteness then dictates to conclude that $N^{j}=0$ for all $j=1, \ldots, k$ and $i=1, \ldots, n$ hence in fact $s^{\prime}$ is holomorphic over the whole $C$ as desired.

Now we turn to the construction of the relevant modular functor. Suppose that $\Sigma \leftrightarrow M$ is a compact surface without boundary. Choose any complex structure $C=C(\Sigma)$ on it and $n$ distinct marked points $p_{1}, \ldots, p_{n} \in C$ given by multiple-points of the immersion. Let $E^{\prime}:=\left.T M \otimes_{\mathbb{R}} \mathbb{C}\right|_{C \backslash\left\{p_{1}, \ldots, p_{n}\right\}}$ be a holomorphic unitary bundle over the punctured surface. Or rather more generally, if $C=\sqcup_{i} C_{i}$ is an abstract compact non-punctured Riemann surface as in Theorem 3.2 with connected components $C_{i}$ then let $E$ be 
a holomorphic unitary bundle over $C$ with $\operatorname{rk}_{\mathbb{C}}\left(\left.E\right|_{C_{i}}\right)=4, \operatorname{deg}\left(\left.E\right|_{C_{i}}\right)=0$ and $\operatorname{dim}_{\mathbb{C}} H^{0}(C ; \mathscr{O}(E))=4$. Then in terms of the restricted bundle $E^{\prime}:=$ $\left.E\right|_{C \backslash\left\{p_{1}, \ldots, p_{n}\right\}}$ our choice is as follows:

(10)

$$
\tau\left(\Sigma, p_{1}, \ldots, p_{n}\right):=\left\{\begin{aligned}
\operatorname{Cliff}\left(H^{0}\left(C \backslash\left\{p_{1}, \ldots, p_{n}\right\} ; \mathscr{O}\left(E^{\prime}\right)\right) \cap L_{l o c}^{2}(C ; E)\right) \\
\quad \text { if }\left(\Sigma, p_{1}, \ldots, p_{n}\right) \neq \emptyset ; \\
\mathbb{C} \quad \text { if }\left(\Sigma, p_{1}, \ldots, p_{n}\right)=\emptyset
\end{aligned}\right.
$$

that is, this vector space is the underlying vector space of the complex Clifford algebra of the scalar product space

$$
\left(H^{0}\left(C \backslash\left\{p_{1}, \ldots, p_{n}\right\} ; \mathscr{O}\left(E^{\prime}\right)\right) \cap L_{\text {loc }}^{2}(C ; E),(\cdot, \cdot)_{L^{2}(C)}\right) \cong \mathbb{C}_{\text {Hermite }}^{4}
$$

consisting of vector fields on $M$ which, upon restriction to $C$, are holomorphic except in the marked points and have locally finite energy.

Lemma 4.2. Let $\left(\Sigma, p_{1}, \ldots, p_{n}\right)$ be a smooth surface with marked points and take a complex structure $C=C(\Sigma)$ rendering it a Riemann surface with marked points $\left(C, p_{1}, \ldots, p_{n}\right)$. Also take the holomorphic unitary bundle $E^{\prime}$ over $C \backslash\left\{p_{1}, \ldots, p_{n}\right\}$ as before. Attach to every marked point $p_{i} \in C$ the single label

$\nu:=\left\{\right.$ a holomorphic section of $E^{\prime}$ has a finite energy singularity in $\left.p_{i} \in C\right\}$.

Then the assignment (9) with the choice (10) is a modular functor which is not normalized in the sense that $\tau\left(S^{2}, \emptyset\right)=\operatorname{Cliff}\left(H^{0}\left(\mathbb{C} P^{1} ; \mathscr{O}\left(E^{\prime}\right)\right)\right)$ instead of $\tau\left(S^{2}, \emptyset\right)=\mathbb{C}$.

Moreover the vector spaces $\tau\left(\Sigma, p_{1}, \ldots, p_{n}\right)$ fit together into a trivial holomorphic vector bundle $\mathscr{E}$ over the coarse moduli space $\mathscr{M}_{g, n}$ of genus $g$ Riemann surfaces with $n$ marked points carrying a flat connection $\nabla$ (the Knizhnik-Zamolodchikov connection). The vector $P_{C, \omega, A} \in H^{0}(C ; \mathscr{O}(E))$ is the value at $C \in \mathscr{M}_{g, n}$ of a section $P_{\Sigma, \omega, A}$ of this bundle over $\mathscr{M}_{g, n}$ satisfying $\nabla P_{\Sigma, \omega, A}=0$.

Proof. We check the three relevant axioms of [1, Definition 5.1.2]. First of all Lemma 4.1 yields that if $\left(\Sigma, p_{1}, \ldots, p_{n}\right) \neq \emptyset$ then

$$
\tau\left(\Sigma, p_{1} \ldots, p_{n}\right) \cong \operatorname{Cliff}\left(H^{0}(C ; \mathscr{O}(E))\right)
$$


consequently the vector spaces are finite dimensional. It also readily follows from (10) that

$\tau\left(\left(\Sigma_{1}, p_{1}, \ldots, p_{n}\right) \sqcup\left(\Sigma_{2}, q_{1}, \ldots, q_{m}\right)\right) \cong \tau\left(\Sigma_{1}, p_{1}, \ldots, p_{n}\right) \otimes_{\mathbb{C}} \tau\left(\Sigma_{2}, q_{1}, \ldots, q_{m}\right)$

as vector spaces, in agreement with [1, part (iii) of Definition 5.1.2]. The second axiom to check is [1, part (iv) of Definition 5.1.2] which is the glueing axiom. Let $\gamma \subset\left(\Sigma, p_{1}, \ldots, p_{n}\right)$ be a closed curve without self-intersections. Cut $\left(\Sigma, p_{1}, \ldots, p_{n}\right)$ along $\gamma$. The resulting surface has naturally the structure of a not necessarily connected punctured surface $\left(\tilde{\Sigma}, p_{1}, \ldots, p_{n}, q_{1}, q_{2}\right)$ where the two new marked points $q_{1}, q_{2}$ come from the circle $\gamma$. Putting $\tilde{E}:=$ $\left.E\right|_{C \backslash\left(\left\{p_{1}, \ldots, p_{n}\right\} \cup \gamma\right)}$ into (10) by the aid of Lemma 4.1 we obtain that locally finite energy meromorphic sections on $\left(\tilde{C}, p_{1}, \ldots, p_{n}, q_{1}, q_{2}\right)$ correspond to those on $\left(C, p_{1}, \ldots, p_{n}\right)$ consequently, taking into account that there is only a single label $\nu$ with its meaning,

$$
\tau\left(\tilde{\Sigma}, p_{1}, \ldots, p_{n}, q_{1}, q_{2}\right) \cong \tau\left(\Sigma, p_{1}, \ldots, p_{n}\right)
$$

hence the glueing axiom holds in a trivial way here.

The third axiom to check is the functorial behaviour under diffeomorphisms [1, part (ii) of Definition 5.1.2]. In turn this is equivalent to checking the existence of a Knizhnik-Zamolodchikov connection. Let $\mathscr{M}_{g, n}$ be the coarse moduli space of connected non-singular Riemann surfaces of genus $g$ and $n$ marked points. We take a complex vector bundle $\mathscr{E}$ over $\mathscr{M}_{g, n}$ whose fibers over $\left(C, p_{1}, \ldots, p_{n}\right) \in \mathscr{M}_{g, n}$ are the individual spaces of conformal blocks $\tau\left(\Sigma, p_{1}, \ldots, p_{n}\right)$ constructed from the holomorphic bundle $E^{\prime}$ over $C \backslash\left\{p_{1}, \ldots, p_{n}\right\}$ or equivalently $E$ over $C$. Recall that $M$ is acted upon by its diffeomorphism group. Hence the subgroup $\operatorname{Diff}_{\Sigma}^{+}(M) \subset \operatorname{Diff}^{+}(M)$ consisting of $\Sigma$-preserving diffeomorphisms acts on the real smooth punctured surface such that it deforms its complex structure i.e., $\left(\Sigma, p_{1}, \ldots, p_{n}\right)$ and $\left(f(\Sigma), f\left(p_{1}\right), \ldots, f\left(p_{n}\right)\right)$ correspond in general to different points in $\mathscr{M}_{g, n}$. This subgroup also acts on $C^{\infty}(C ; E)$ by pullback. Consequently it transforms the subspaces

$$
\tau\left(\Sigma, p_{1}, \ldots, p_{n}\right) \cong \operatorname{Cliff}\left(H^{0}(C ; \mathscr{O}(E))\right) \subset \operatorname{Cliff}\left(C^{\infty}(\Sigma ; E)\right)
$$

giving rise to linear isomorphisms

$$
\tau\left(\Sigma, p_{1}, \ldots, p_{n}\right) \cong \tau\left(f(\Sigma), f\left(p_{1}\right), \ldots, f\left(p_{n}\right)\right) \quad \text { for all } f \in \operatorname{Diff}_{\Sigma}^{+}(M) .
$$

These linear isomorphisms can be interpreted as parallel translations along $\mathscr{E}$ by a flat connection $\nabla$ called the Knizhnik-Zamolodchikov connection. Note 
that since the representation of $\Sigma$-preserving diffeomorphisms on $C^{\infty}(\Sigma ; E)$ is not only projective but in fact a true representation, the resulting connection is not only projectively but truely flat on $\mathscr{E}$. In particular the bundle $\mathscr{E}$ as a complex vector bundle is trivial over $\mathscr{M}_{g, n}$ but is equipped with a holomorphic structure. Via Lemma 4.1 the holomorhic section $P_{C, \omega, A} \in$ $H^{0}(C ; \mathscr{O}(E))$ can be regarded as a meromorphic one i.e., $P_{C, \omega, A} \in H^{0}(C \backslash$ $\left.\left\{p_{1}, \ldots, p_{n}\right\} ; \mathscr{O}\left(E^{\prime}\right)\right)$. Define a section $P_{\Sigma, \omega, A}$ of $\mathscr{E}$ on $\mathscr{M}_{g, n}$ by putting $P_{\Sigma, \omega, A}(C):=P_{C, \omega, A}$. It follows from the invariance of the definition (7) of the quasilocal energy-momentum 4 -vector

$$
\begin{aligned}
P_{C, \omega, A} & \in H^{0}(C ; \mathscr{O}(E)) \subset \operatorname{Cliff}\left(H^{0}(C ; \mathscr{O}(E))\right) \\
& \cong \operatorname{Cliff}\left(H^{0}\left(C \backslash\left\{p_{1}, \ldots, p_{n}\right\} ; \mathscr{O}\left(E^{\prime}\right)\right) \cap L_{l o c}^{2}(C ; E)\right)=\tau\left(\Sigma, p_{1}, \ldots, p_{n}\right)
\end{aligned}
$$

under diffeomorphisms that as the complex structure varies $P_{\Sigma, \omega, A}$ of $\mathscr{E}$ satisfies $\nabla P_{\Sigma, \omega, A}=0$ i.e., is parallel for the Knizhnik-Zamolodchikov connection.

We conclude that the assignment (9) with (10) is a $C$-extended modular functor as in [1, Definition 5.1.2] i.e., a weakly conformal field theory á la G. Segal [16].

After having constructed the modular functor, we find the vector space on which it acts hence exhibit the conformal field theory given by (9) and (10). This step is very simple: the space $\left(\Sigma, p_{1}, \ldots, p_{n}\right)$ identified with an oriented smooth cobordism between the disconnected compact oriented 1manifolds $S_{p_{1}}^{1} \sqcup \cdots \sqcup S_{p_{k}}^{1}$ and $S_{p_{k+1}}^{1} \sqcup \cdots \sqcup S_{p_{n}}^{1}$. To the oriented 1-manifold $S_{p_{1}}^{1} \sqcup \cdots \sqcup S_{p_{k}}^{1} \sqcup\left(S_{p_{k+1}}^{1}\right)^{*} \sqcup \cdots \sqcup\left(S_{p_{n}}^{1}\right)^{*}$, regardless what it actually is, we associate the finite dimensional complex vector space $S \otimes_{\mathbb{C}} S^{*}$ where $S$ is the unique irreducible complex Clifford-module of $\tau\left(\Sigma, p_{1}, \ldots, p_{n}\right)$. The resulting conformal field theory is a topological one because its state space is finite dimensional and its correlation functions are insensitive for the insertion of marked points (due to Lemma 4.1).

\section{References}

[1] B. Bakalov and A. Kirillov Jr., Lectures on tensor categories and modular functors, Providence, American Mathematical Society, (2001).

[2] R. Brunetti, K. Fredenhagen, and K. Rejzner, Quantum gravity from the point of view of locally covariant quantum field theory, preprint, (2013), 51pp. arXiv:1306.1058 [math-phys]. 
[3] R. Brunetti, K. Fredenhagen, and R. Verch, The generally covariant locality principle - a new paradigm for local quantum physics, Comm. Math. Phys. 237 (2003), 31-68.

[4] F. Cardone and R. Mignani, Lorentz invariance breakdown: a brief survey, in: Deformed spacetimes, Fundamental Theories of Physics 157, 189-193, Springer, New York, (2007).

[5] D. M. DeTurck, Existence of metrics with prescribed curvature: local theory, Invent. Math. 65 (1981), 179-207.

[6] S. K. Donaldson and P. B. Kronheimer, The geometry of four-manifolds, Clarendon Press, Oxford, (1990).

[7] A. J. Dougan and L. J. Mason, Quasilocal mass constructions with positive energy, Phys. Rev. Lett. 67 (1991), 2119-2122.

[8] M. H. Freedman, The topology of four-manifolds, Journ. Diff. Geom. 17 (1982), 357-454.

[9] J. Gasqui, Sur la résolubilité locale des équations d'Einstein, Compositio Math. 47 (1982), 43-69.

[10] R. Haag, Local quantum physics, Springer-Verlag, Berlin, (1993).

[11] G. S. Hall, Curvature collineations and determination of the metric from the curvature in general relativity, Gen. Rel. Grav. 15 (1983), 581-589.

[12] R. Hedrich, String theory - nomological unification and the epicycles of the quantum field theory paradigm, preprint, (2011), 23pp. arXiv: 1101.0690 [physics].

[13] S. Hollands and R. M. Wald, Quantum field theory in curved spacetime, preprint, (2014), 52pp. arXiv:1401.2026v1 [gr-qc].

[14] O. Kowalski, On regular curvature structures, Math. Z. 125 (1972), 129-138.

[15] F. Riesz and B. Szőkefalvi-Nagy, Leçons d'analyse fonctionnelle, Gauthier-Villars, Paris, Akadémiai Kiadó, Budapest, (1965).

[16] G. B. Segal, Two-dimensional conformal field theories and modular functors, IXth International Congress of Mathematical Physics (Swansea, 1988), Hilger, Bristol, 22-37, (1989).

[17] I. M. Singer and J. A. Thorpe, The curvature of 4-dimensional Einstein spaces, in: Global analysis, Papers in honour of K. Kodaira, 355-365, Princeton Univ. Press, Princeton, (1969). 
[18] L. B. Szabados, Quasi-local energy momentum and angular momentum in general relativity, Living Rev. Relativity 12 (2009), 4.

[19] M. E. Taylor, Partial differential equations I-III, AMS 115, 116, 117, Springer, New York, $(1996,1996,1996)$.

[20] K. K. Uhlenbeck, Removable singularities in Yang-Mills fields, Comm. Math. Phys. 83 (1982), 11-29.

[21] E. Witten, Quantum field theory and the Jones polynomial, Comm. Math. Phys. 121 (1989), 351-399.

Department of Geometry, Mathematical Institute, Faculty of Science Budapest University of TeChNOLOGY AND ECONOMiCs

Egry J. U. 1, H ÉP., H-1111 Budapest, Hungary

E-mail address: etesi@math.bme.hu 